\title{
Pollen morphology of the genus Euonymus (Celastraceae)
}

\author{
O. A. Gavrilova ${ }^{1}$, D. A. Britski ${ }^{1}$, V. V. Grigorieva1, V. F. Tarasevich ${ }^{1}$, A. E. Pozhidaev¹, V. M. Leunova² \\ ${ }^{1}$ Komarov Botanical Institute RAS, Prof. Popov St., 2, St. Petersburg, 197376, Russia.E-mail: olgaangav@gmail.com \\ ${ }^{2}$ Moscow State University, Leninskye gory, 1-12, Moscow, 119234,Russia.E-mail: cedrus@rambler.ru
}

Keywords: aperture, exine structure, microscopy, sporoderm, systematics.

Summary. Pollen of 62 species from the genus Euonymus (Celastraceae) were studied using light (LM), confocal laser scanning (CLSM) and scanning electron (SEM) microscopes. This is approximately half of the species composition of the taxon. The genus, as a whole family, is quite homogeneous by palynomorphological characteristics and represented by 3 -colporate pollen type. On the other hand, some species and (or) group of species could be segregated considering the details of structure (aperture structures, exine ornamentation). We have found 3-colporate pollen in all investigated taxa and 19 species also have 2- and/or 4-6-deviated grains with different colpi arrangement. Their structure has been studied in more detail with a CLSM 3-dimentional reconstruction. Grains are isopolar, ellipsoidal or spheroidal medium sized, from 17 to $41 \mu \mathrm{m}$ in diameter. Colpi are long, spired at the ends. Endoaperture (ora) range from poorly-defined, rounded or slightly elongared along the colpus up to well-defined, with sharp edges, elliptical and elongared along the equator. LM-observing shows H-shaped aperture; CLSM data demonstrate that they are constructed from ora bridge and exine thinning on both sides of parallel colpus. The exine is tectate, columellate, with well-defined columella layer. Exine thickness is from 1.5 to $5.1 \mu \mathrm{m}$. The ornamentation varies from reticulate to microperforate. The species groups divided according to their palynomorphological characteristics generally do not coincide with the genus subdivision onto subgenera and sections. The taxonomic significance of various palynological characteristics is discussed.

\section{Морфология пыльцы представителей рода Euonymus (Celastraceae)}

\author{
О. А. Гаврилова ${ }^{1}$, Д. А. Брицкий ${ }^{1}$, В. В. Григорьева ${ }^{1}$, В. Ф. Тарасевич ${ }^{1}$, \\ А. Е. Пожидаев ${ }^{1}$, В. М. Леунова ${ }^{2}$ \\ ${ }^{1}$ Ботанический институт им. В. Л. Комарова РАН, ул. Проф. Попова, д. 2, г. Санкт-Петербург, 197376, Россия \\ ${ }^{2}$ Московский государственный университет, Ленинские горы, 1-12, г. Москва, 119234, Россия
}

Ключевые слова: апертуры, микроскопия, систематика, спородерма, структура экзины.

Аннотация. С помощью светового (СМ), конфокального лазерного сканирующего (КСЛМ) и сканирующего электронного (СЭМ) микроскопов изучена пыльца 62 видов рода Euоnymus (Celastraceae). Род, как и все семейство, довольно однороден по палиноморфологическим признакам и представлен трехбороздно-оровым типом пыльцевых зерен. Однако некоторые виды и (или) группы видов могут быть выделены с учетом деталей строения (апертур или поверхности экзины). У всех исследованных таксонов обнаружены 3-бороздно-оровые пыльцевые зерна, у 19 представителей наряду с 3-бороздно-оровыми встречаются также 2- и/или 4-6-бороздно-оровые зерна с разным расположением борозд и ор. Структура апертур изучена более подробно с использованием трехмерных реконструкций конфокальной микроскопии. Зерна радиально-симметричные, эллипсоидальные или сфероидальные, среднего размера, от 17 до 41 мкм в диаметре. Борозды длинные, заостренные на концах. Эндоапертуры (оры) встречаются от слабо очерченных, неясных, округлых или чуть вытянутых по борозде до хорошо различимых, с четкими краями, эллипсоидальных, вытянутых по экватору. Наблюдение 
с помощью СМ показывает Н-образные апертуры, данные КЛСМ демонстрируют, что они состоят из оровой перемычки и утоньшений экзины с двух сторон параллельно бороздам. Экзина столбиковая, тектатная, в большинстве случаев хорошо выражен столбиковый слой. Толщина экзины от 15 до 5,1 мкм. Поверхность варьирует от крупносетчатой до микроперфорированной. Выделенные по палиноморфологическим характеристикам группы видов в основном не совпадают с подразделениями рода на подроды и секции. Обсуждается таксономическое значение отдельных палинологических признаков.

The genus Euonymus L. belongs to the family Celastraceae R. Br. of the order Celastrales and contains from 129 (Ma, 2001) to 200 species (Leonova, 1974; Tzvelev, 2004; Savinov, Baikov, 2007), which are deciduous and evergreen shrubs or small trees. Plants of Euonymus grow in the undergrowth or along broad-leaved, mixed or coniferous forests in tropical, subtropical and temperate areas of both hemispheres. Because of the beautifully colored seeds, spindle trees are often cultivated as ornamental plants in gardens and parks all around the world.

The most of systematics (Leonova, 1974; Ma, 2001; Tzvelev, 2004; Savinov, Baikov, 2007) accept a genus after R. A. Blakelock (1951): the subgenus Kalonymus R. Beck which was allocated by Ya. I. Prokhanov $(1949,1960)$ to separate genus, and the genera allocated by T. Nakai (1941) as sections are included in Euonymus. Now, there are two main worldwide taxonomic systems of the genus: 1) the R. A. Blakelock system (1951) (including with T. Leonova (1974) changes which have made many taxonomical decisions of T. Nakai (1941) and Th. Loesener (1942)), and 2) the J.-S. Ma (2001) system. According to R. A. Blakelock (1951) the genus includes 6 sections in subgenus Euonymus and one section in subgenus Kalonymus. T. Leonova (1974), and after her, I. Savinov and K. Baikov (2007), I. Savinov (2007) have divided the genus into 12 sections, 10 of them are from subgenus Euonymus and 2 of them are from Kalonymus. After J.-S. Ma (2001) the genus includes 5 sections, 4 of them are combined into subgenus Euonymus, and all sections are allocated on the basis of fruits structure. J.-S. Ma (2001) has united three sections of R. A. Blakelock (1951): Biloculares Rouy et Fouc., Multiovulares Loes., Stenocarpus Blakel. in one section Euonymus, while the section Melanocarya (Turcz.) Nakai, Echinococcus Nakai and Ilicifolia Nakai have been left almost invariable, although the number of species has been reduced. The subgenus Kalonymus is acknowledged as the youngest group of spindle trees (Shimanovich, 1987; Savinov, Baikov, 2007). N. Tzvelev (2004) doesn't divide a genus into subgenera and has united spindle trees of Eastern Europe into 8 sections, having allocated one new section Nanevonymus with one species $E$. nanus. B. Schulz (2006) has described fruits and seeds of 30 widely cultivated species of spindle trees from Central Europe and species' criteria have been discussed. Based on the morphological and molecular data, M. P. Simmons et al. (2012) considered that all infrageneric classifications weren't natural and demanded rather significant revision. Y.-N. Li et al. (2014) concluded that only two sections (Echinococcus and Kalonymus) of J.-S. Ma (2001) system correspond to molecular groups.

The pollen morphology of the genus has been insufficiently studied, and also pollen data hasn't been used for taxonomy and systematics. There are palynomorphological characteristics of 26 Euonymus species, which were obtained mainly with light microscope (LM), rarely scanning electron (SEM) microscope (Erdtman, 1952; Ikuse, 1956; Wang et al., 1960; Nair, 1965; Lobreau, 1969; Heusser, 1971; Huang, 1972; Kupriyanova, 1972; Shimakura, 1973; Poliychuk, 1978; Nakamura, 1980; Artyushenko, Romanova, 1984; Chester, Raine, 2001; Premathilake, Nilsson, 2001; Perveen, Qaiser, 2008). D. Lobreau-Callen and B. Lugardon (1972-1973) have been studied sporoderm ultrastructure of 2 species (E. europaeus L., E. japonicus Thunb.) using SEM and transmission electron (TEM) microscope.

In our paper, the pollen of 62 species (specimens) from the genus Euonymus (Celastraceae) was studied using LM and SEM and partly with confocal laser scanning (CSLM) microscopes. This is about a half of the species' composition of the taxon.

The aim of our research work was the study of pollen morphology of the genus Euonymus by SEM and CLSM and supplement LM data. We tried to specify features of a structure and variety of pollen characteristics, that expand our knowledge of this taxon, and to estimate the value of the pollen morphological characteristics for taxonomy.

\section{Material and methods}

Pollen samples were collected in herbarium of the Komarov Botanical Institute, Russian Academy of Sciences (St. Petersburg, Russia) (BIN RAS). The following vouchers were investigated: E. acan- 
thocarpus Franch. (E. acanthocarpus Franch. var. sutchuenensis Franch. ex Diels): "China 19071909, s. n.”; E. actinocarpus Loes. (E. angustatus Spraque): "Hong Kong, Shek-O, Y. W. Tsam 1178. 24 V 1940"; E. alatus (Thunb.) Siebold: "Sakhalin, South-Sakhalin reservation, S. Kravchenko s. n. 22 VI 1950"; E. americanus L.: "Baltimore, North Carolina, № 422в. 26 V 1894”; E. artropurpureus Jacq.: "Texas, Houston, A. Travers 323. 24 V 1957"; E. benthamii Lundell (E. acuminatus Benth.): "Mexico, Benth. № 452, 1870"; E. bungeanus Maxim.: "Manchuria, Dalniy, J. Vasilieva, s. n. 1903"; E. cornutus Hemsl.: "China, Western Szechuan, E. H. Wilson 1047. 07 VIII 1907-1909”; E. czernjaevii Klokov: "USSR, Donetsk, E. Mordak, L. Sveschnicova s. n. 02 V 1977"; E. dasydictyon Loes. et Rehder: "Tibet, highlands between the stations of O-Lun-shi and Makhchuk. 18 V 1893"; E. dichotomus Heyne ex Wall.: "India orientalis. Herb. Wight. 484"; E. echinatus Wall.: "Nepal, Bagmati zone, Kathmandu District Sheopuri Ridge, D. N. Nicolson 3345. 13 V 1967"; E. elegantissimus Loes. et Rehder: "China, Peking, W. Y. Hsia s. n. 15 IV 1926"; E. europaeus L. "Belorussia, Gomel, N. Kozlovskaya s. n. 23 V 1962"; E. fimbriatus Wall.: "N. W. Himalaya, Deota Temple, D. Dultne 22545, 09 V 1989"; E. frigidus Wall.: "Sikkim, Sunducrophro, C. B. Clarke 35672a. 04 VI 1884"; E. gibber Hance (E. miyakey Hayata): "China, Hainan, S. K. Lau s. n. 18 V 1933; E. glaber Roxb.: "Calcutta s. n."; E. grandiflorus Wall.: "Chao Cim ssu im Kreise Teng fong, Honan, A. K. Schingler s. n., VIII 1907”; E. hamiltanianus Wall.: "Cult. in garden, Kew, R. A. Blakelork. 06 X 1977"; E. hederacea Champ. ex Benth. (E. radicans Miq.): "Suchumi, T. Leonova s. n. 05 VI 1955"; E. integerrimus Prokh.: "China, prov. Gansu, dist. Piplan, M. P. Petrov s. n. 10 VI 1957; E. japonicus Thunb.: "Crimea, Alupka, K. K. Kossinskiy s. n. 1911"; E. juzepczukii Leon.: "Forest flora of Siwalik and Jaunsar Deobau divisions. Mohammed Yusuf R. 32. 25 V 1923"; E. lanceifolius Loes.: "China, Western Szechuan, E. H. Wilson. 06-11 VIII 1907-1909”; E. latifolius (L.) Mill.: "Crimea, Mangup, N. N. Tzvelev, D. B. Geltman, N. A. Medvedeva, G. A. Mustafina 692. 18 V 1984"; E. laxiflorus Champ. ex Benth. (E. paniculatus Wight ex Lawson): "Imperial Forest college, Dehra Doon, Indu Bhushan Maitra B. Sc. Roll., № 1. IV 1924"; E. maackii Rupr.: "South-Usury distr., N. V. Schipchinskaya 227. 15 VI 1913"; E. macropterus Rupr.: "Kaliningrad, E. Pobedimova 52, 09 V 1950"; E. maximomowiczianus Prokh.: "Kedrovaya pad reservation, V. N. Vasiliev, E. V. Volko- va, L. I. Ivanina s. n. 5 VI 1950"; E. melananthus Franch. et Sav.: "Japan, Oita, Naoiri-gun, Kujumachi, Mt. Kuro-dake, Shinya Masyda 101072, 27 V 1989"; E. miniatus Tolm.: "Sachalin, Chechov, F. M. Chernyaeva s. n. 13 VI 1963"; E. moldavicus Klokov: "Russia, Voronezh distr. 30 IV 1930"; E. myrianthus Hemsl.: "Cult. Royal Bot. Garden Kew, R. A. Blakelork. 11 V 1944"; E. nanus M. Bieb.: "Ukraine, Vinnica distr., s. n. 03 VI 1928"; E. nitidus Benth. (E. chinensis Lindl.): "Hohg Kong, Shin Ying Hu 5222. 04 V 1968"; E. obovatus Nutt.: "Michigan (U.S.A.), Frere Rolland - German № 6443. 17 V 1956"; E. occidentalis Nutt. ex Torr. "Washington, Richard R. Halse 4040. 21 VI 1990"; E. oxyphyllus Miq.: "Sapporo, S. Galeenobii 2567. VI 1882"; E. patens Rehder: "Log. Kiang-si, Lu-shan. VIII 1934"; E. pauciflorus Maxim.: "Russia, Vladivostok, E. Pobedimova, G. Konovalov 70. 05 VI 1959"; E. phellomanus Loes.: "China, Sothwestern Kansu, J. F. Rock 12822. VII 1925"; E. pendulus Wall.: "Western Himalaya, prov. Simla, № 486, $20 \mathrm{~V}$ 1856"; E. planipes Koehne: "Sakhalin, South-Sakhalin reservation, S. Kravchenko s. n. 22 VI 1950"; E. porphyreus Loes.: "China, Western Szechuan, E. H. Wilson 968. 06 VIII 1908"; E. przewalskii Maxim.: "South-western Kansu, J. F. Rock s. n. VII 1925"; E. sachalinensis (F. Schmidt) Maxim.: "South Sachalin, M. G. Popov, K. D. Stepanova s. n. 16 VI 1950"; E. sacrosanctus Koidz: "Primorsky region, Nikolsk, M. Fritz s. n. 1912"; E. salicifolius Loes.: "China, Yunnan, № 11718a; E. sanguineus Loes.: "Western Hupeh, E. H. Wilson 3111. 05 VII 1907-1909"; E. semenovii Regel et Herder: "Almaty, Flora Illiensis, 1886"; E. sieboldianus Blume: "Sachalin, s. n."; E. subtriflorus Blume: "Japonia insula Jesso, circa Hakodate, Dr. Albrecht s. n. 1861-1863"; E. szechuanensis Chen H. Wang: "China, Szechuan, Dr. Aug. Henry 5562, III 1889; E. theifolius Wall.: "China, Yunnan, A. Henry 10544"; E. tingens Wall.: "Imperial Forest College, place of collection Deoban, Indu Bhusman Maitra B. Sc., № 19. V 1924”; E. velutinus Fisch. et C. A. Mey.: "Herb. Bunze, Riga, Albursgebing Talysh geb. 28 VIII 1847"; E. verrucosus Scop.: "Baschkortostan, Ufa, B. A. Fedchenko, 19. 15 VI 1926"; E. vidalii Franch. et Sav.: "Japan, Yokohama, Maximowicz 1221. 06-18 V 1862"; E. yakushimensis Makino: "Japan, Kagoshima, T. Yahara, S. Akiyama s. n. 14 VII 1987”; E. yedoensis Koehne var. koehneana Loes.: "China, western Hupeh, E. H. Wilson 353, 06-10 VII 1907-1909"; E. yesoensis Koidz.: "Kuril isl., Schikotan, E. Pobedimova, G. Konovalov 701. 11 VII 1959". 
Pollen was acetolysed according to G. Erdtman (1952). Unstained grains were mounted in glycerin jelly and were sealed with paraffin. LM studies were conducted in the laboratory of palynology in BIN RAS. Pollen grains have been studied with SEM JEOL JSM-6390 (SEM) and with an LSM 780 (CLSM) at the Core Centrum 'Cell and Molecular Technologies in Plant Science' in BIN RAS. CLSM investigation was after O. Gavrilova (2014). The palynological terminology follows W. Punt et al. (2007) and M. Hesse et al. (2009).

\section{Results}

Pollen grains of all investigated species are 3-colporate, medium sized, from 17 to $41 \mu \mathrm{m}$ in diameter, with tectate or semitectate, columellate exine, perforate or reticulate types of ornamentation.

Morphological and morphometrical pollen characteristics are presented in Tables 1 and 2. The species are presented according to subgenera and sections of J.-S. Ma (2001) system, the species, recognized in that system, are in bold, the other names are listed as synonyms.

General pollen morphology: the form and sizes. Pollen of all species is radially symmetric, from prolate or prolate spheroidal to oblate or oblate spheroidal (Fig. 1, 4). Grains in polar view are from 3-4-lobate (Fig. 1: 1, 6; Fig. 4: 8, 11, 12, 15) to circular (Fig. 1: 2,5). Only pollen of 4 species E. gibber, E. japonicus, E. laxiflorus, E. nitidus are 3-angular (Fig. 1: 3, 4; Fig. 4: 1) in polar view. Pollen equatorial view are round or elliptic. Polar axis and equatorial diameter varies from 17 to $41 \mu \mathrm{m}$.

Table 1

Morphological and morphometrical characteristics of pollen grains of species from the subgenus Euonymus of genus Euonymus (Celastraceae)

\begin{tabular}{|c|c|c|c|c|c|}
\hline Species & $\begin{array}{l}\text { Pollen size (polar } \\
\text { axis } \mathrm{x} \text { equatorial } \\
\text { axis, } \mu \mathrm{m} \text { ) and } \\
\text { pollen grains form }\end{array}$ & $\begin{array}{l}\text { The number of } \\
\text { colpi, their features, } \\
\% \text { of deviated } \\
\text { grains }\end{array}$ & $\begin{array}{l}\text { Endoapertures - } \\
\text { Ora, their features } \\
\text { and diameter, } \mu \mathrm{m}\end{array}$ & $\begin{array}{l}\text { Ornamentation, } \\
\text { their features, } \\
\text { lumina, } \mu \mathrm{m},(\text { width } \\
\text { of muri, } \mu \mathrm{m})\end{array}$ & $\begin{array}{c}\text { exine } \\
\text { thickness, } \\
\qquad \mu \mathrm{m}\end{array}$ \\
\hline \multicolumn{6}{|c|}{ 2. Echinococcus } \\
\hline E. acanthocarpus & $\begin{array}{c}33-36 \times 28-30 \\
\text { oblate spheroidal }\end{array}$ & $\begin{array}{l}3-(4)-(6)-(1), \\
\text { syncolpi, } 5 \%\end{array}$ & $\begin{array}{c}\text { round, H-shaped, } \\
5-6\end{array}$ & $\begin{array}{c}\text { reticulate, } \\
\text { heterobronchate, } \\
0.2 \text { and } 0.5-1.4 \\
(0.4)\end{array}$ & $3.4-3.6$ \\
\hline E. actinocarpus & $\begin{array}{c}27-29 \times 25-27 \\
\text { oblate spheroidal }\end{array}$ & 3 & $\begin{array}{c}\text { round or } \\
\text { quadrangular, H- } \\
\text { shaped, } 5\end{array}$ & $\begin{array}{c}\text { reticulate, } \\
\text { heterobronchate, } \\
0.3 \text { and } 0.4-0.6 \text { to } \\
1.2(0.4)\end{array}$ & $3.0-3.5$ \\
\hline E. americanus & $\begin{array}{l}30-31 \times 27-31 \\
\text { prolate spheroidal } \\
\text { to oblate spheroidal }\end{array}$ & 3 & $\begin{array}{l}\text { not clear, } \mathrm{H}- \\
\text { shaped, } 3\end{array}$ & $\begin{array}{c}\text { reticulate- } \\
\text { perforate, } 0.2-1.2 \\
(0.7-1.1)\end{array}$ & $2,4-3,2$ \\
\hline E. echinatus & $\begin{array}{c}27-32 \times 25-35(40) \\
\text { oblate spheroidal }\end{array}$ & $\begin{array}{c}3-(4)-(6) \\
11 \%\end{array}$ & not clear, 3 & $\begin{array}{c}\text { reticulate, } 0.8 \text { up to } \\
1.7(0.2-0.3)\end{array}$ & $2.3-3.6$ \\
\hline E. obovatus & $\begin{array}{l}25-30 \times 25-29 \\
\text { oblate spheroidal }\end{array}$ & 3 & round, 4 & $\begin{array}{c}\text { reticulate, } \\
\text { heterobronchate, } \\
0.1-0.5 \text { and } 0.7-1.5 \\
(0.2)\end{array}$ & $3.5-3.8$ \\
\hline \multicolumn{6}{|c|}{ 3. Melanocarya } \\
\hline E. alatus & $\begin{array}{c}31-35 \times 26-30 \\
\text { prolate spheroidal } \\
\text { to oblate spheroidal }\end{array}$ & 3 & not clear, 3-4 & $\begin{array}{l}\text { reticulate, } 1-3 \\
(0.5-0.7)\end{array}$ & $3.5-4.4$ \\
\hline E. pendulus & $\begin{array}{c}28-30 \times 26-30 \\
\text { oblate spheroidal }\end{array}$ & $\begin{array}{c}3-(2)-(1) \\
7 \%\end{array}$ & $\begin{array}{c}\text { round, H-shaped, } \\
5-6\end{array}$ & $\begin{array}{c}\text { reticulate, } \\
\text { heterobronchate, } \\
0.2-0.5 \text { and } 0.6-1.2 \\
(0.3)\end{array}$ & $2.7-3.0$ \\
\hline E. sacrosanctus & $\begin{array}{c}31-35 \times 27-30 \\
\text { prolate spheroidal }\end{array}$ & $\begin{array}{c}3-(4)-(6) \\
20 \% \\
\end{array}$ & $\begin{array}{c}\text { round, not clear, } \\
3-4 \\
\end{array}$ & $\begin{array}{l}\text { reticulate, } 0.8 \text { up to } \\
2.0(0.4)\end{array}$ & $3.0-4.5$ \\
\hline E. subtriflorus & $\begin{array}{c}22-30 \times 20-25 \\
\text { prolate spheroidal } \\
\text { to oblate spheroidal }\end{array}$ & 3 & $\begin{array}{l}\text { round or oval, not } \\
\text { clear, 5-6 }\end{array}$ & $\begin{array}{l}\text { reticulate, } 1.0 \text { up to } \\
2.5(0.3)\end{array}$ & $2.5-3.1$ \\
\hline \multicolumn{6}{|c|}{ 4. Ilicifolia } \\
\hline E. benthamii & $\begin{array}{c}28-33 \times(25) 28-30 \\
\text { oblate spheroidal }\end{array}$ & 3 & $\begin{array}{c}\text { round, not clear, } \\
5-6\end{array}$ & $\begin{array}{c}\text { reticulate, } 0.4-1.2 \\
(0.5)\end{array}$ & $2.7-3.7$ \\
\hline
\end{tabular}


Continuation of table 1

\begin{tabular}{|c|c|c|c|c|c|}
\hline Species & $\begin{array}{l}\text { Pollen size (polar } \\
\text { axis x equatorial } \\
\text { axis, } \mu \mathrm{m} \text { ) and } \\
\text { pollen grains form }\end{array}$ & $\begin{array}{l}\text { The number of } \\
\text { colpi, their features, } \\
\% \text { of deviated } \\
\text { grains }\end{array}$ & $\begin{array}{l}\text { Endoapertures - } \\
\text { Ora, their features } \\
\text { and diameter, } \mu \mathrm{m}\end{array}$ & $\begin{array}{c}\text { Ornamentation, } \\
\text { their features, } \\
\text { lumina, } \mu \mathrm{m},(\text { width } \\
\text { of muri, } \mu \mathrm{m})\end{array}$ & $\begin{array}{l}\text { exine } \\
\text { thickness, } \\
\mu \mathrm{m}\end{array}$ \\
\hline E. hederacea & $\begin{array}{c}25-28 \times 25-27 \\
\text { oblate spheroidal }\end{array}$ & 3 & $\begin{array}{l}\text { equatorial elon- } \\
\text { gated, H-shaped, } \\
\text { not clear, small- } \\
\text { sized, 1-2 }\end{array}$ & $\begin{array}{c}\text { reticulate, } \\
\text { heterobronchate, } \\
0.5-0.9 \text { up to } \\
1.3 \text { and } 0.1-0.2 \\
(0.4-0.5)\end{array}$ & $2.3-2.6$ \\
\hline E. japonicus & $\begin{array}{c}22-25 \times 20-24 \\
\text { prolate spheroidal } \\
\text { to oblate spheroidal }\end{array}$ & $3-(2), 1 \%$ & $\begin{array}{c}\text { round, small-sized, } \\
\text { H-shaped, } 2\end{array}$ & $\begin{array}{c}\text { reticulate- } \\
\text { perforate, } 0,1 \text { up to } \\
1.0(0,3 \text { up to } 1)\end{array}$ & $2.0-3.5$ \\
\hline E. patens & $\begin{array}{c}29-30 \times 29-32 \\
\text { oblate spheroidal }\end{array}$ & 3 & $\begin{array}{c}\text { H-shaped, not } \\
\text { clear, small-sized, } \\
1\end{array}$ & $\begin{array}{c}\text { reticulate, } \\
\text { heterobronchate, } \\
0.1-0.2 \text { and } 0.6-1.0 \\
(0.3)\end{array}$ & $3.0-3.4$ \\
\hline E. theifolius & $\begin{array}{c}26-28 \times 25-28 \\
\text { prolate spheroidal }\end{array}$ & $\begin{array}{c}\text { 3-(4), W-shaped } \\
\text { colpi, } 10 \%\end{array}$ & $\begin{array}{l}\text { H-shaped, not } \\
\text { clear, round, } 3\end{array}$ & $\begin{array}{c}\text { reticulate, } \\
\text { heterobronchate, } \\
0.1 \text { and } 0.5-1.3 \\
(0,3)\end{array}$ & $2.0-2.8$ \\
\hline E. yakushimensis & $\begin{array}{c}30-35 \times 27-33,5 \\
\text { prolate spheroidal } \\
\text { to oblate spheroidal }\end{array}$ & 3 & $\begin{array}{c}\text { H-shaped, round, } \\
5-6\end{array}$ & $\begin{array}{c}\text { reticulate- } \\
\text { perforate, } 0.25 \text { up } \\
\text { to } 1.2(0.3 \text { up to } \\
1.0)\end{array}$ & 3.2 \\
\hline \multicolumn{6}{|c|}{ 5. Euonymus } \\
\hline E. artropurpureus & $\begin{array}{c}24-25 \times 22-25 \\
\text { oblate spheroidal }\end{array}$ & 3 & $\begin{array}{c}\text { round, H-shaped, } \\
2-5\end{array}$ & $\begin{array}{c}\text { reticulate, } 0.7 \text { up to } \\
3.0(0.4-0.5)\end{array}$ & $2.2-2.7$ \\
\hline E. bungeanus & $\begin{array}{c}29-31 \times 27-28 \\
\text { oblate spheroidal }\end{array}$ & 3 & $\begin{array}{c}\text { round, } 4-5, \mathrm{H}- \\
\text { shaped }\end{array}$ & $\begin{array}{c}\text { reticulate, } \\
\text { heterobronchate, } \\
0.7-2.1 \text { and } 0.2-0.3 \\
(0.4)\end{array}$ & $3.0-3.5$ \\
\hline E. czernjaevii & $\begin{array}{c}24-27 \times 23-26 \\
\text { prolate spheroidal } \\
\text { to oblate spheroidal }\end{array}$ & 3 & not clear, 3 & $\begin{array}{c}\text { reticulate, } \\
\text { heterobronchate, } \\
0.1-0.2 \text { up to } 0.3- \\
1.0(0.3)\end{array}$ & $2.2-2.5$ \\
\hline E. dichotomus & $\begin{array}{c}24-27 \times 23-25 \\
\text { prolate spheroidal } \\
\text { to oblate spheroidal }\end{array}$ & 3 & $\begin{array}{c}\text { round, small-sized, } \\
1.5\end{array}$ & $\begin{array}{c}\text { (micro)reticulate, } \\
0.1-0.3(0.5)\end{array}$ & $2.3-3.0$ \\
\hline E. europaeus & $\begin{array}{c}28-30 \times 26-30 \\
\text { prolate spheroidal } \\
\text { to oblate spheroidal }\end{array}$ & 3 & round, not clear, 3 & $\begin{array}{l}\text { reticulate, hetero- } \\
\text { bronchate, } 0.8 \text { up } \\
\text { to } 1.8 \text { and } 0.2-0.5 \\
(0.5)\end{array}$ & $2.3-2.9$ \\
\hline E. gibber & $\begin{array}{c}24-29 \times 25-30 \\
\text { oblate spheroidal }\end{array}$ & 3 & $\begin{array}{c}\text { equatorial } \\
\text { elongated, 5-7 }\end{array}$ & $\begin{array}{c}\text { reticulate, hetero- } \\
\text { bronchate, } 0.2 \text { up to } \\
1.3(0.4-0.8)\end{array}$ & 2.3 \\
\hline E. glaber & $\begin{array}{c}21-29 \times 20-26 \\
\text { prolate spheroidal } \\
\text { to oblate spheroidal }\end{array}$ & 3 & $\begin{array}{l}\text { not clear, small- } \\
\text { sized, } 1\end{array}$ & $\begin{array}{c}\text { reticulate- } \\
\text { perforate, } 0.1 \text { up to } \\
0.8(0.4)\end{array}$ & $1.4-2.0$ \\
\hline E. grandiflorus & $\begin{array}{c}\text { 34-38 } \times 32-35 \\
\text { prolate spheroidal } \\
\text { to oblate spheroidal }\end{array}$ & $3-(4)-(6), 50 \%$ & $\begin{array}{l}\text { H-shaped, small- } \\
\text { sized, not clear, 1-2 }\end{array}$ & $\begin{array}{l}\text { reticulate, } 0.6-0.7 \\
\text { to } 1.7(0.4)\end{array}$ & $3.5-3.7$ \\
\hline E. hamiltonianus & $\begin{array}{c}28-30 \times 25-29 \\
\text { prolate spheroidal } \\
\text { to oblate spheroidal }\end{array}$ & $3-(2), 2 \%$ & $\begin{array}{c}\text { round or equatorial } \\
\text { alongated, not } \\
\text { clear, } 3-4, \mathrm{H}- \\
\text { shaped } \\
\end{array}$ & $\begin{array}{c}\text { reticulate, } 1.0 \text { up } \\
\text { to } 2.2 \text { and } 0.2-0.5 \\
(0.2)\end{array}$ & $2.4-3.5$ \\
\hline E. integerrimus & $\begin{array}{c}30-34 \times 30-37 \\
\text { oblate to oblate } \\
\text { spheroidal }\end{array}$ & $3-(4)-(6), 1 \%$ & $\begin{array}{l}\text { not clear, small- } \\
\text { sized, 1-2 }\end{array}$ & $\begin{array}{l}\text { reticulate, } 0.4-0.8 \\
\text { up to } 1.3(0.5)\end{array}$ & $3.4-3.5$ \\
\hline E. juzepczukii & $\begin{array}{c}25-28 \times 23-28 \\
\text { prolate spheroidal } \\
\text { to oblate spheroidal }\end{array}$ & 3 & round, 4 & $\begin{array}{c}\text { reticulate, } 1.0-1.5 \\
(0.2)\end{array}$ & 2.0 \\
\hline
\end{tabular}


Continuation of table 1

\begin{tabular}{|c|c|c|c|c|c|}
\hline Species & $\begin{array}{l}\text { Pollen size (polar } \\
\text { axis x equatorial } \\
\text { axis, } \mu \mathrm{m} \text { ) and } \\
\text { pollen grains form }\end{array}$ & $\begin{array}{l}\text { The number of } \\
\text { colpi, their features, } \\
\% \text { of deviated } \\
\text { grains }\end{array}$ & $\begin{array}{l}\text { Endoapertures - } \\
\text { Ora, their features } \\
\text { and diameter, } \mu \mathrm{m}\end{array}$ & $\begin{array}{c}\text { Ornamentation, } \\
\text { their features, } \\
\text { lumina, } \mu \mathrm{m},(\text { width } \\
\text { of muri, } \mu \mathrm{m})\end{array}$ & $\begin{array}{l}\text { exine } \\
\text { thickness, } \\
\qquad \mu \mathrm{m}\end{array}$ \\
\hline E. lanceifolius & $\begin{array}{c}29-32 \times 27-28 \\
\text { prolate spheroidal }\end{array}$ & 3 & not clear, 2 & $\begin{array}{l}\text { reticulate, } 0.6-1.2 \\
\text { up to } 2.0(0.3)\end{array}$ & $3.5-4.0$ \\
\hline E. laxiflorus & $\begin{array}{c}23-26 \times 26-29 \\
\text { oblate spheroidal }\end{array}$ & $\begin{array}{c}3-(1)-(2) \\
11 \%\end{array}$ & round, clear, 3 & $\begin{array}{l}\text { reticulate, } 0.1-0.7 \\
\text { up to } 1.5(0.6-0.7)\end{array}$ & 2.0 \\
\hline E. maackii & $\begin{array}{c}28-30 \times 25-31 \\
\text { oblate spheroidal }\end{array}$ & 3 & $\begin{array}{c}\text { H-shaped, round, } \\
4-6\end{array}$ & $\begin{array}{c}\text { reticulate, } \\
\text { heterobronchate, } \\
0.2-0.3 \text { and } 0.6-2.0 \\
(0.3)\end{array}$ & $2.7-3.1$ \\
\hline E. melananthus & $\begin{array}{l}33-35 \times 29-33 \\
\text { oblate spheroidal }\end{array}$ & 3 & $\begin{array}{c}\text { round, not clear, } \\
3-4\end{array}$ & $\begin{array}{l}\text { reticulate, }(0.6) \\
1.0-2.0 \text { up to } 27 \\
(0.3)\end{array}$ & $3.5-4.0$ \\
\hline E. moldavicus & $\begin{array}{c}27-29 \times 25-27 \\
\text { oblate spheroidal }\end{array}$ & 3 & round, 3 & $\begin{array}{c}\text { reticulate, } 0.5 \text { up to } \\
1.0(0.4-0.5)\end{array}$ & $2.5-3.1$ \\
\hline E. myrianthus & $\begin{array}{c}21-23 \times 22-25 \\
\text { oblate spheroidal }\end{array}$ & 3 & $\begin{array}{l}\text { small-sized, not } \\
\text { clear, 1-2 }\end{array}$ & $\begin{array}{c}\text { perforate, } 0.2-0.5 \\
(0.2-0.9)\end{array}$ & $1.8-2.0$ \\
\hline E. nanus & $\begin{array}{c}21-25 \times 20-23 \\
\text { prolate spheroidal } \\
\text { to oblate spheroidal }\end{array}$ & 3 & round, oval, 3-4 & $\begin{array}{c}\text { reticulate, } \\
\text { heterobronchate, } \\
0.1-0.2 \text { and } 0.3-1.0 \\
(0.5)\end{array}$ & $3.9-4.8$ \\
\hline E. nitidus & $\begin{array}{c}17-20 \times 18-22 \\
\text { oblate spheroidal }\end{array}$ & 3 & $\begin{array}{l}\text { round, } \mathrm{H} \text {-shaped, } \\
\text { not clear, 2-3 }\end{array}$ & $\begin{array}{l}\text { perforate, } 0.1-0.4 \text {. } \\
(0.3-0.8)\end{array}$ & 2.3 \\
\hline E. occidentalis & $\begin{array}{c}31-35 \times 31-40 \\
\text { oblate spheroidal }\end{array}$ & 3 & $\begin{array}{l}\text { not clear, round, H- } \\
\text { shaped, } 3\end{array}$ & $\begin{array}{c}\text { reticulate, } 0.5-1.2 \\
(0.2)\end{array}$ & $3.2-4.0$ \\
\hline E. pauciflorus & $\begin{array}{c}27-32 \times 23-29 \\
\text { prolate spheroidal } \\
\text { to oblate spheroidal }\end{array}$ & $\begin{array}{l}3-(4)-(6), W- \\
\text { shaped, } 20 \%\end{array}$ & $\begin{array}{c}\text { round, not clear, } \\
3-4\end{array}$ & $\begin{array}{c}\text { reticulate, } 0.5 \text { up to } \\
2.0(0.3)\end{array}$ & $2.2-4.0$ \\
\hline E. phellomanus & $\begin{array}{c}32-35 \times 28-32 \\
\text { oblate spheroidal }\end{array}$ & 3 & $\begin{array}{l}\text { round, not clear, } \\
4-5\end{array}$ & $\begin{array}{c}\text { reticulate, } \\
\text { heterobronchate, } \\
0.5-0.8 \text { up to } 2.0 \\
(0.3) \\
\end{array}$ & $3.0-4.0$ \\
\hline E. przewalskii & $\begin{array}{c}27-30 \times 25-28 \\
\text { oblate spheroidal }\end{array}$ & 3 & $\begin{array}{c}\text { round, H-shaped, } \\
4-5\end{array}$ & $\begin{array}{c}\text { reticulate, } \\
\text { heterobronchate, } \\
0.2-0.3 \text { and } 0.4-1.2 \\
(0.4)\end{array}$ & $2.9-3.3$ \\
\hline E. salicifolius & $\begin{array}{c}24-30 \times 28-32 \\
\text { oblate to oblate } \\
\text { spheroidal }\end{array}$ & 3 & $\begin{array}{c}\text { round, } \mathrm{H}-\text { shaped, } \\
2-4\end{array}$ & $\begin{array}{c}\text { reticulate, } \\
\text { heterobronchate, } \\
0.1-0.2 \text { and } 0.5-1.5 \\
(0.5)\end{array}$ & $2.0-2.7$ \\
\hline E. semenovii & $\begin{array}{c}25-28 \times 22-27 \\
\text { prolate spheroidal } \\
\text { to oblate spheroidal }\end{array}$ & 3 & $\begin{array}{l}\text { oval, equatorial } \\
\text { elongated, } 4\end{array}$ & $\begin{array}{c}\text { reticulate, } \\
\text { heterobronchate, } \\
0.2-0.3 \text { and } 0.5-1.5 \\
(0.2)\end{array}$ & $2.0-3.8$ \\
\hline E. sieboldianus & $\begin{array}{c}30-34 \times 29-31 \\
\text { prolate spheroidal } \\
\text { to oblate spheroidal }\end{array}$ & $3-4-(6), 2 \%$ & $\begin{array}{l}\text { round, equatorial } \\
\text { elongated, } 5\end{array}$ & $\begin{array}{c}\text { reticulate, } 0.5 \text { up to } \\
2.2 .(0.4)\end{array}$ & $2.2-3.0$ \\
\hline E. tingens & $\begin{array}{c}27-31 \times 28-31 \\
\text { oblate spheroidal }\end{array}$ & $3-(4), 9 \%$ & $\begin{array}{l}\text { equatorial } \\
\text { elongated, not } \\
\text { clear, 4-6 }\end{array}$ & $\begin{array}{l}\text { reticulate, hetero- } \\
\text { bronchate, } 0.2 \text { and } \\
0.5-1.4(0.6-0.7)\end{array}$ & 2.3 \\
\hline E. velutinus & $\begin{array}{c}25-35 \times 22-33 \\
\text { prolate spheroidal } \\
\text { to oblate spheroidal }\end{array}$ & 3 & round, clear, 3-4 & $\begin{array}{l}\text { reticulate, } 1.0-1.8 \\
(0.2)\end{array}$ & $2.0-3.8$ \\
\hline E. verrucosus & $\begin{array}{c}27-29 \times 25-30 \\
\text { prolate spheroidal } \\
\text { to oblate spheroidal }\end{array}$ & 3 & $\begin{array}{c}\text { round, H-shaped, } \\
3-4\end{array}$ & $\begin{array}{c}\text { reticulate, } 0.5-1.5 \\
(0.4)\end{array}$ & 3.0 \\
\hline
\end{tabular}


End of table 1

\begin{tabular}{|l|c|c|c|c|c|}
\hline \multicolumn{1}{|c|}{ Species } & $\begin{array}{c}\text { Pollen size (polar } \\
\text { axis } \mathrm{x} \text { equatorial } \\
\text { axis, } \mu \mathrm{m}) \text { and } \\
\text { pollen grains form }\end{array}$ & $\begin{array}{c}\text { The number of } \\
\text { colpi, their features, } \\
\text { \% of deviated } \\
\text { grains }\end{array}$ & $\begin{array}{c}\text { Endoapertures }- \\
\text { Ora, their features } \\
\text { and diameter, } \mu \mathrm{m}\end{array}$ & $\begin{array}{c}\text { Ornamentation, } \\
\text { their features, } \\
\text { lumina, } \mu \mathrm{m},(\text { width } \\
\text { of muri, } \mu \mathrm{m})\end{array}$ & $\begin{array}{c}\text { exine } \\
\text { thickness, } \\
\mu \mathrm{m}\end{array}$ \\
\hline E. vidalii & $\begin{array}{c}30-35 \times 27-33 \\
\text { oblate spheroidal }\end{array}$ & 3 & round, 5 & $\begin{array}{c}\text { reticulate- } \\
\text { perforate, } 1.0-1.8 \\
\text { up to 2.5 }(0.5-1.3)\end{array}$ & 3.2 \\
\hline $\begin{array}{l}\text { E. yedoensis } \text { var. } \\
\text { koehneana }\end{array}$ & $\begin{array}{c}32-38 \times 32-35 \\
\text { oblate spheroidal }\end{array}$ & 3 & $\begin{array}{c}\text { not clear, equatorial } \\
\text { alongated, 4-7 }\end{array}$ & $\begin{array}{c}\text { reticulate, } \\
\text { heterobronchate, } \\
0.2-0.3 \text { and } 0.4-1.6 \\
(0.3-0.7)\end{array}$ & 4.2 \\
\hline
\end{tabular}

Table 2

Morphological and morphometrical characteristics of pollen grains of species from the subgenus Kalonymus of genus Euonymus (Celastraceae)

\begin{tabular}{|c|c|c|c|c|c|}
\hline Species & $\begin{array}{l}\text { Pollen size (polar axis } \mathrm{x} \\
\text { equatorial axis, } \mu \mathrm{m} \text { ) and } \\
\text { pollen grains form }\end{array}$ & $\begin{array}{l}\text { The number } \\
\text { of colpi, } \% \\
\text { of deviated } \\
\text { grains }\end{array}$ & $\begin{array}{c}\text { Endoapertures - Ora, } \\
\text { their features and } \\
\text { diameter, } \mu \mathrm{m}\end{array}$ & $\begin{array}{l}\text { Ornamentation, their } \\
\text { features, lumina, } \mu \mathrm{m} \\
\text { (width of muri, } \mu \mathrm{m} \text { ) }\end{array}$ & $\begin{array}{c}\text { exine } \\
\text { thickness, } \\
\mu \mathrm{m}\end{array}$ \\
\hline E. cornutus & $\begin{array}{l}29-32 \times 27-30 \\
\text { oblate spheroidal }\end{array}$ & $3-(2), 1 \%$ & $\begin{array}{c}\text { Round or } \\
\text { quadrangular, H- } \\
\text { shaped, } 2-4\end{array}$ & $\begin{array}{c}\text { reticulate, } \\
\text { heterobronchate, } 0.7- \\
1.7 \text { and } 0.3-0.4(0.4)\end{array}$ & $3.4-3.5$ \\
\hline E. dasydictyon & $\begin{array}{c}31-37 \times 25-31 \\
\text { oblate spheroidal }\end{array}$ & 3 & $\begin{array}{c}\text { not clear, small-sized, } \\
2\end{array}$ & $\begin{array}{l}\text { reticulate, } 0.7-1.5 \text { up } \\
\text { to } 1.8 .(0.4)\end{array}$ & $3.4-4.0$ \\
\hline E. elegantissimus & $\begin{array}{c}35-37 \times 33-37 \\
\text { oblate spheroidal } \\
\end{array}$ & 3 & not clear, 3 & $\begin{array}{l}\text { reticulate, } 0.5-0.9 \text { up } \\
\text { to } 2.0(0.5)\end{array}$ & $3.5-4.0$ \\
\hline E. fimbricatus & $\begin{array}{c}25-30 \times 23-30 \text { prolate } \\
\text { spheroidal to oblate } \\
\text { spheroidal } \\
\end{array}$ & $3-(2), \leq 1 \%$ & $\begin{array}{l}\text { not clear, round, } \\
\text { small-sized, } 2\end{array}$ & $\begin{array}{l}\text { reticulate, } 0.5 \text { up to } \\
2.0 .(0.3)\end{array}$ & $2.0-3,2$ \\
\hline E. frigidus & $\begin{array}{l}23-24 \times 23-25 \text { oblate to } \\
\text { oblate spheroidal }\end{array}$ & 3 & $\begin{array}{c}\text { not clear, small-sized, } \\
1\end{array}$ & $\begin{array}{c}\text { verrucate-reticulate, } \\
\text { non-mesurable }\end{array}$ & $1.8-2.1$ \\
\hline E. latifolius & $\begin{array}{c}38-41 \times 30-41 \text { prolate } \\
\text { spheroidal to oblate } \\
\text { spheroidal }\end{array}$ & $\begin{array}{l}\text { 3-(4)-(6), } \\
\text { 39\%: }\end{array}$ & round, not clear, 5 & $\begin{array}{c}\text { reticulate, } \\
\text { heterobronchate, } 0.5 \text { up } \\
\text { to } 3 \text { and } 0.2-0.3 \cdot(0.5)\end{array}$ & $4.3-5.7$ \\
\hline E. macropterus & $\begin{array}{c}32-34 \times 30-35 \text { prolate } \\
\text { spheroidal to oblate } \\
\text { spheroidal }\end{array}$ & 3 & $\begin{array}{c}\text { round not clear, 2-3, } \\
\text { H-shaped }\end{array}$ & $\begin{array}{l}\text { reticulate, hetero- } \\
\text { bronchate, } 1.2 \text { up to } 2.7 \\
\text { and } 0.3(0.5-0.7)\end{array}$ & $2.0-3.1$ \\
\hline $\begin{array}{l}\text { E. maximomo- } \\
\text { wiczianus }\end{array}$ & $\begin{array}{c}30-32 \times 25-30 \text { prolate } \\
\text { spheroidal to oblate } \\
\text { spheroidal }\end{array}$ & $3-(4), \leq 1 \%$ & $\begin{array}{c}\text { not clear, small-sized, } \\
1\end{array}$ & $\begin{array}{c}\text { reticulate, } 0.5 \text { up to } 2.0 \\
(0.2)\end{array}$ & $3.9-4.8$ \\
\hline E. miniatus & $\begin{array}{c}30-37 \times 31-38 \text { oblate } \\
\text { spheroidal }\end{array}$ & 3 & round, not clear, 3 & $\begin{array}{c}\text { reticulate, } 1.0 \text { up to } 3.0 \\
(0.4-0.5)\end{array}$ & $3.0-4.0$ \\
\hline E. oxyphyllus & $\begin{array}{c}31-38 \times 29-37 \text { oblate } \\
\text { spheroidal }\end{array}$ & 3 & not clear, round, 5 & $\begin{array}{c}\text { reticulate, } 1.0-2.5 . \\
(0.2)\end{array}$ & $3.9-5.1$. \\
\hline E. planipes & $\begin{array}{c}31-35 \times 29-32 \text { prolate } \\
\text { spheroidal to oblate } \\
\text { spheroidal }\end{array}$ & $\begin{array}{c}3-(2)-(1) \\
4 \%\end{array}$ & $\begin{array}{l}\text { not clear, oval or } \\
\text { round, } 3\end{array}$ & $\begin{array}{l}\text { reticulate, } \\
\text { heterobronchate, } 1.0- \\
\text { 2.0. }(0.2) \\
\end{array}$ & $2.5-4.8$ \\
\hline E. porphyreus & $\begin{array}{c}31-34 \times 30-33 \text { prolate } \\
\text { spheroidal to oblate } \\
\text { spheroidal }\end{array}$ & 3 & not clear, 5-6 & $\begin{array}{c}\text { reticulate, } \\
\text { heterobronchate, } 0.6- \\
1.2 \text { up to } 2.3 \\
\end{array}$ & $4.0-4.3$ \\
\hline E. sachalinensis & $\begin{array}{c}31-35 \times 31-33 \text { oblate } \\
\text { spheroidal } \\
\end{array}$ & 3 & round, not clear, 3 & $\begin{array}{c}\text { reticulate, } 1.0 \text { up to } 3.0 \\
(0.6)\end{array}$ & $3.0-4.3$ \\
\hline E. sanguineus & $\begin{array}{c}31-33 \times 27-35 \text { prolate } \\
\text { spheroidal to oblate } \\
\text { spheroidal }\end{array}$ & $3-(2), 4 \%$ & $\begin{array}{l}\text { round or equatorial } \\
\text { elongated, not clear, } 4\end{array}$ & $\begin{array}{l}\text { reticulate, } 0.5 \text { up to } 2.0 \\
(0.3-0.4)\end{array}$ & $2.0-2.7$ \\
\hline E. szechuanensis & $\begin{array}{c}30-32 \times 25-31 \text { oblate } \\
\text { spheroidal }\end{array}$ & 3 & $\begin{array}{l}\text { round or equatorial } \\
\text { elongated, } 5\end{array}$ & $\begin{array}{c}\text { reticulate, } 0.8 \text { up to } 2.0 \\
(0.4-0.5)\end{array}$ & $3.0-4.5$ \\
\hline E. yesoensis & $\begin{array}{c}30-32 \times 29-34 \text { prolate } \\
\text { spheroidal to oblate } \\
\text { spheroidal }\end{array}$ & $3-(2), 1.5 \%$ & H-shaped, round, 5 & $\begin{array}{c}\text { reticulate-perforate, } \\
0.2-1.5(0.5-0.9)\end{array}$ & $1.5-1.8$ \\
\hline
\end{tabular}


Apertures. All examined species have compound pollen apertures and its grains belong to 3-colporate apertural type. The colpi are long, often wide, with arrow-headed, pointed (Fig. 1, 4), and colpi membranes are granulate, with small, round granules. The ends of colpi sometimes merge in apocolpium and syncolporate grains have been found (Fig. 1: 5).

The number of colpi varies, grains with different number and positions of ecto- and endoapertures have been revealed in some specimens. This phenomenon has been called pollen heteromorphism (Nadot el al.,
2000). We found 2-colporate or 1-syncolporate (Fig. 3: 7, 8; Fig.4: 4, 5, 10) pollen, with W-like colpi band in pairs (Fig. 4: 13, 14), 4-colporate (Fig. 4: $13,14,15), 3$-colporate-3-rugate and 4-colporate-4rugate (Fig. 3: 1, 5, 6, 9; Fig. 4: 11, 12) pollen.

The application of CLSM and 3D-reconstruction of separate grains allows us to specify the disposition of ecto-and endoapertures in deviate pollen forms. The last two types of the deviations (3-colporate3-rugate and 4-colporate-4-rugate) have three or four meridonal colpi (or parts of colpi), starting in one grain pole, but do not reach the other; colpi join
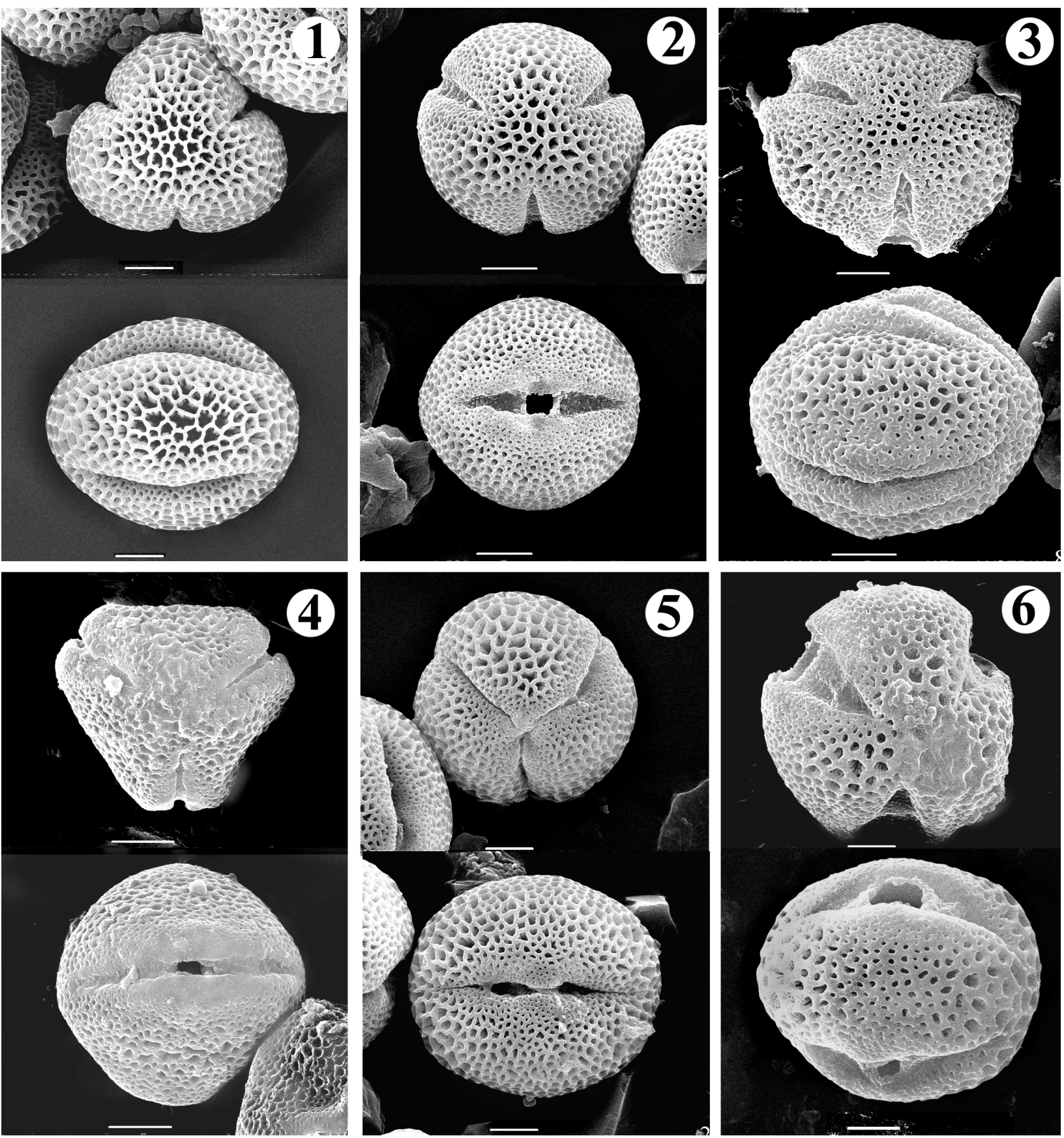

Fig. 1. Polar view and equatorial view of the pollen of Euonymus species (SEM): $1-$ E. melananthus; $2-E$. hederaceus; 3 -E. gibber; $4-$ E. japonicas; $5-$ E. acanthocarpus; $6-E$. vidalii. Scale bar $-5 \mu \mathrm{m}$. 
together near the other pole by three or four rugas, which are perpendicular to colpi. The ora (3 or 4 respectively) are placed in the center of colpi. The percentage of deviant forms can be in amounts of up to $50 \%$ of grains in various Euonymus species.

Nearly one half of species from the subgenus Kalonymus (7 from 16) and near a fourth (quarter) of species from the subgenus Euonymus (13 from 47) show the occurrence of atypical pollen forms. The sections Echinococcus, Melanocarya, Ilicifolia demonstrate many species with pollen heteromorphism, but, in the section Euonymus, it has been found that only 6 specimens have deviated pollen grains from 31 investigated species.

Among the representatives of the subgenus Kalonymus 1-2-colporate deviated pollen forms are more common (5 species), and 4-6-apertural grains appear rarely ( 2 species). The representatives of subgenus Euonymus often have 4-colporate or 3-colporate-3-rugate and 4-colporate-4-rugate atypical grains (7 species), and rarely have 1-2-colporate (5 species), E. acanthocarpus has all types of deviant forms. The percentage of deviant pollen grains among the representatives of the genus
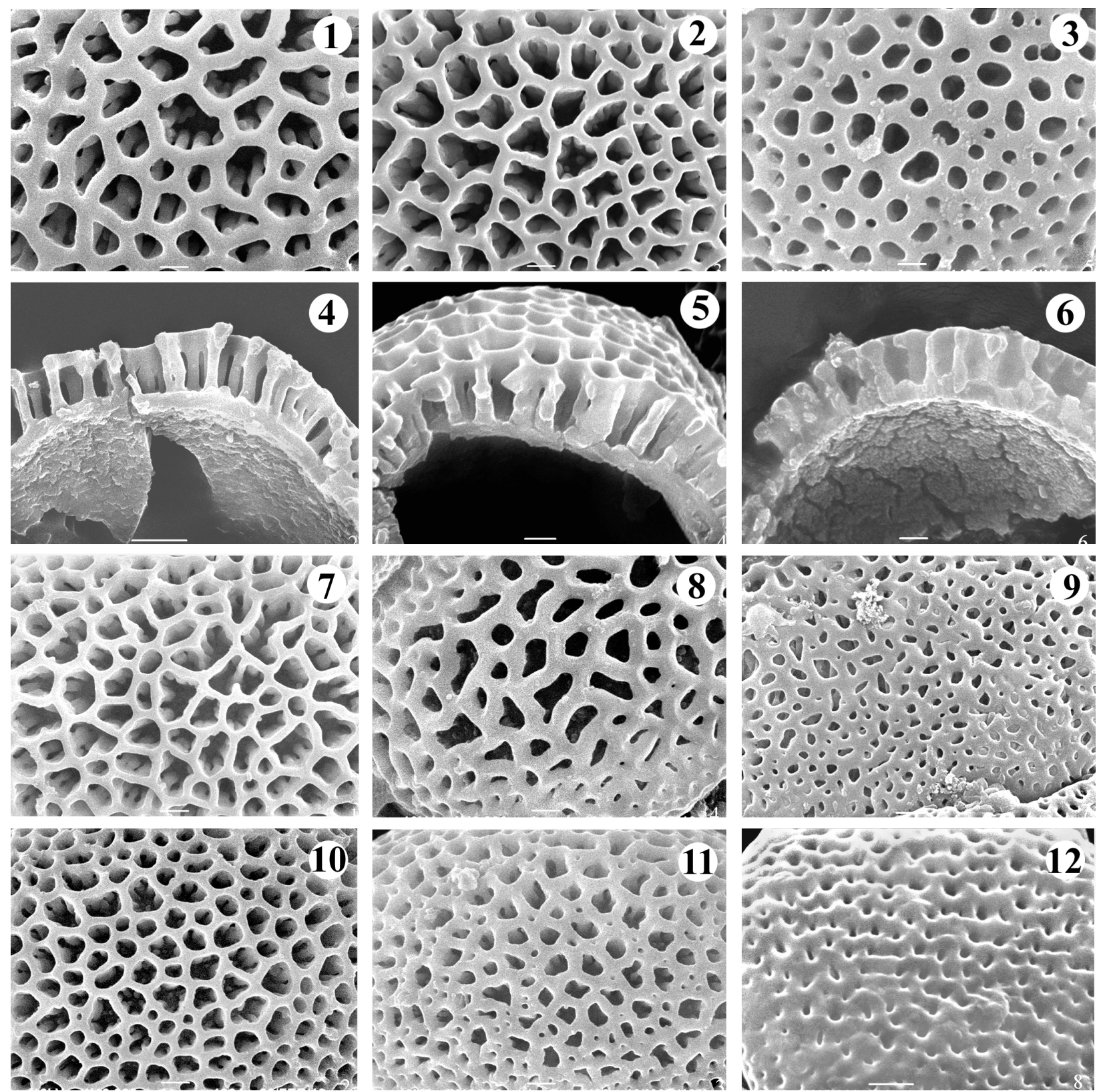

Fig. 2. Pollen ornamentation (1-3, 7-12) and sporoderm cleavage (4-6) of Euonymus species (SEM): 1, 4 - E. porphyreus; 2, $5-$ E. acanthocarpus; 3, 6-E. americanus; $7-$ E. phellomanus; $8-$ E. laxiflorus; $9-$ E. glaber; $10-$ E. pendulus; $11-$ E. nanus; $12-$ E. nitidus. Scale bar $-1 \mu \mathrm{m}$. 
Euonymus varies from individual pollen grains up to $11 \%$ into specimen, but the percentage of atypical grains in E. sacrosanctus and E. pauciflorus reaches $20 \%$, E. latifolius has $39 \%$ and E. grandiflorus possess $50 \%$ of deviated pollen forms.

Endoapertures have been revealed and are seen as obscure, not clear circumscribed, slightly outlined (Fig. 5: 11, 12), with the diameter no more than colpi wideness or up to clear, round, oval, oblong or quadrangular (Fig. 5: 5,6), extending along the equator, which are sometimes significantly exceed the colpi width and pointed at the ends (Fig. 5: 8, 9).

$\mathrm{H}$-shaped apertures formed by ora and exine thinning side by side to colpi (Fig. 4: 6, 9; Fig. 5) appear in pollen of many Euonymus species. CLSM optical sections confirmed the exine thinning are on two sides of the colpi (Fig. 5: 4, 7, 10), which are visible in transmitted light (Fig. 4: 6, 9; Fig. 5: 5, 8, 11). This thinning sometimes is a little longer than the colpi length, but in some cases, they are side by side to the entire colpus and connect to each other on the pole, which are shown at SEM micrographs (Fig. 5: 13 ). The most distinct H-shaped ora are detected in the pollen of $1 / 3$ part of the species from the subgenus Euonymus (16 of 47) and only of 2 species from the subgenus Kalonymus.

Ornamentation. Under a light microscope the pollen sculpture is usually reticulate, lumina specific by different sizes and shapes. Texture varies from pointed to reticulate (Fig. 4).
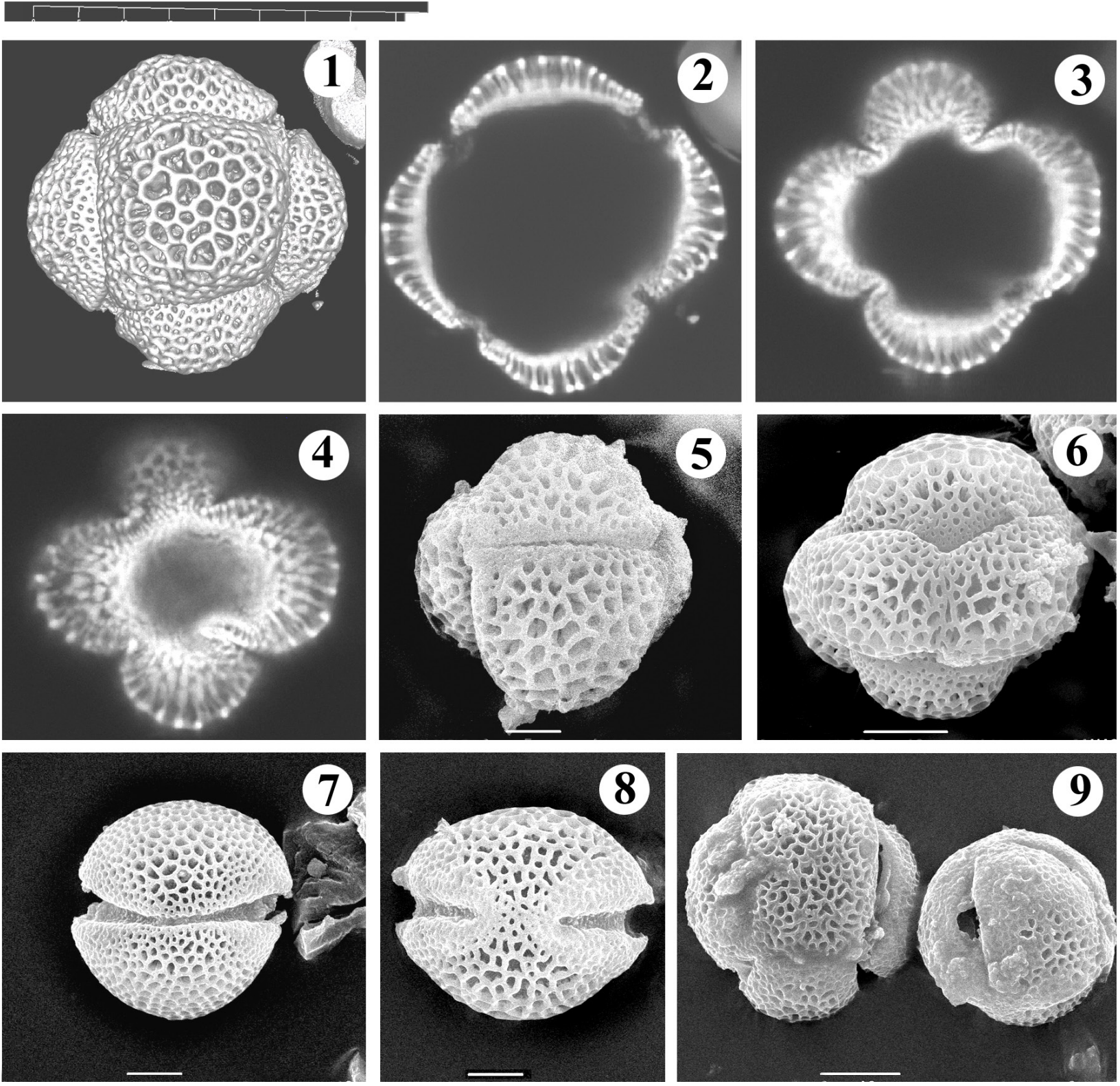

Fig. 3. General view and virtual sections through sporoderm of the pollen of Euonymus species (1-4 - CLSM, 5-9 SEM): 1-5-E. latifolius; $6-$ E. pauciflorus; $7-$ E. pendulus; $8-$ E. laxiflorus; $9-$ E. echinatus. Scale bar $-5 \mu \mathrm{m}$. 
By SEM (Fig. 2) we found reticulate (the lumina glowski, Punt, (1973), Punt et al. (2007), Hesse et wider than $1 \mu \mathrm{m}$, sometimes with big-sized lumina al., 2009) ornamentation. The brochus (mesh of a wider than $2.0 \mu \mathrm{m}$ ) or microreticulate (the lumina reticulum consisting of one lumen and the adjoining and muri smaller than $1 \mu \mathrm{m}$ ), perforate (holes less half of the muri-Erdtman (1952), Punt et al. (2007), than muri and less than $1 \mu \mathrm{m}$ in diameter - Pra- Hesse et al. (2009)) vary in size and contour, and


12
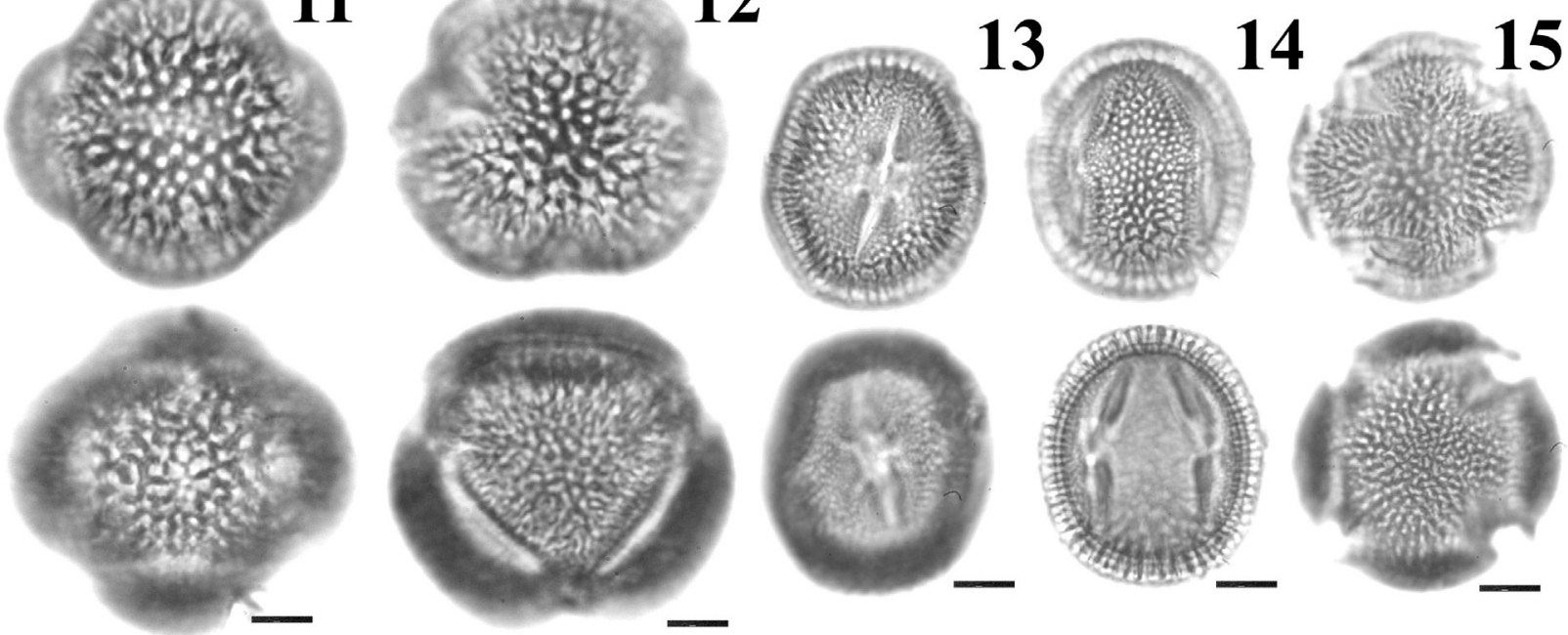

Fig. 4. Polar view $(1-5,8,11,12,15)$ and equatorial view $(6,7,9,10,13,14)$ of the pollen of Euonymus species (LM): 1-7-E. laxiflorus; 8-10-E. pendulus; 11-12-E. acanthocarpus; 13-15-E. grandifloras. Scale bar - $10 \mu \mathrm{m}$. 

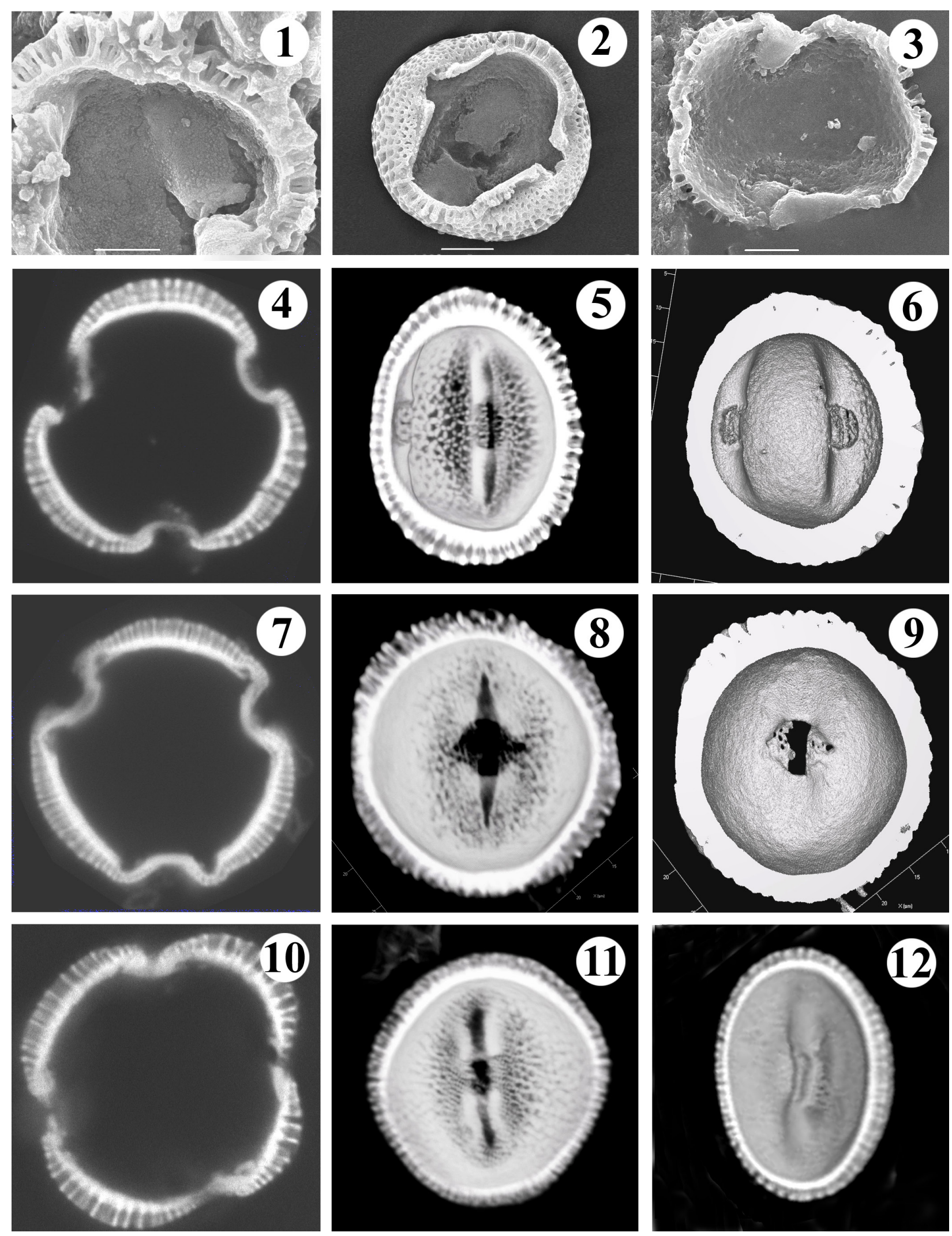

Fig. 5. Sporoderm cleavage (1-3), virtual sections through sporoderm $(4,7,10)$ and reconstructed grains $(5,6,8,9,11$, 12) of the pollen of Euonymus species (1-3-SEM, 4-12 - CLSM: 4, 7, 10 - optical sections; 5, 8, 11, 12 - transmissional mode; 6, 9 - surface mode): 1 - E. macropterus; 2 -E. cornutus; 3, 11 -E. theifolius; 4 -E. europaeus; 5, 6 E. latifolius; $7-$ E. patens; $8,9-$ E. szechuanensis; $10-$ E. echinatus; $12-$ E. glaber. Scale bar $-5 \mu \mathrm{m}$. 
there are heterobronchate surfaces with lumina at different sizes (Fig. 2: 3, 11). The lumina has round, angular, elongated or oblong, rarely oval or irregular outline, its sizes range from smaller than $0.1 \mu \mathrm{m}$ up to $3.5 \mu \mathrm{m}$ in diameter. The width of muri is $0.2-0.8$ $\mu \mathrm{m}$, and rarely riches up to $1.3 \mu \mathrm{m}$. The distance between the perforations is usually $0.2-0.9 \mu \mathrm{m}$. The muri are smooth, direct, rare flexuose or ridge like. The columellae and the bottom of the mesh are visible sometimes, free standing columellae are found sometimes (Fig. 2: 1, 2, 7, 10). Along the colpi the size of lumina decreases, sometimes near the colpi surface it becomes perforated, only in some species (E. benthamii, E. dichotomus, E. frigidus, E. myrianthus, E. nitidus, E. obovatus, E. occidentalis, E. planipes, E. salicifoliaus, E. yakushimensis) the mesh reduction is not revealed. On apocolpium the lumina is usually less or equal to mesocolpium lumina size; on the other hand, only the species E. glaber, E. yedoensis var. koehneana and E. vidalii (Fig. 1: 6) showed an increase in the size of lumina on apocolpium compared with mesosolpium.

Exine. The exine is semitectate or tectate, columellate, from 1.5 to $5.1 \mu \mathrm{m}$ in thickness (Fig. 2:
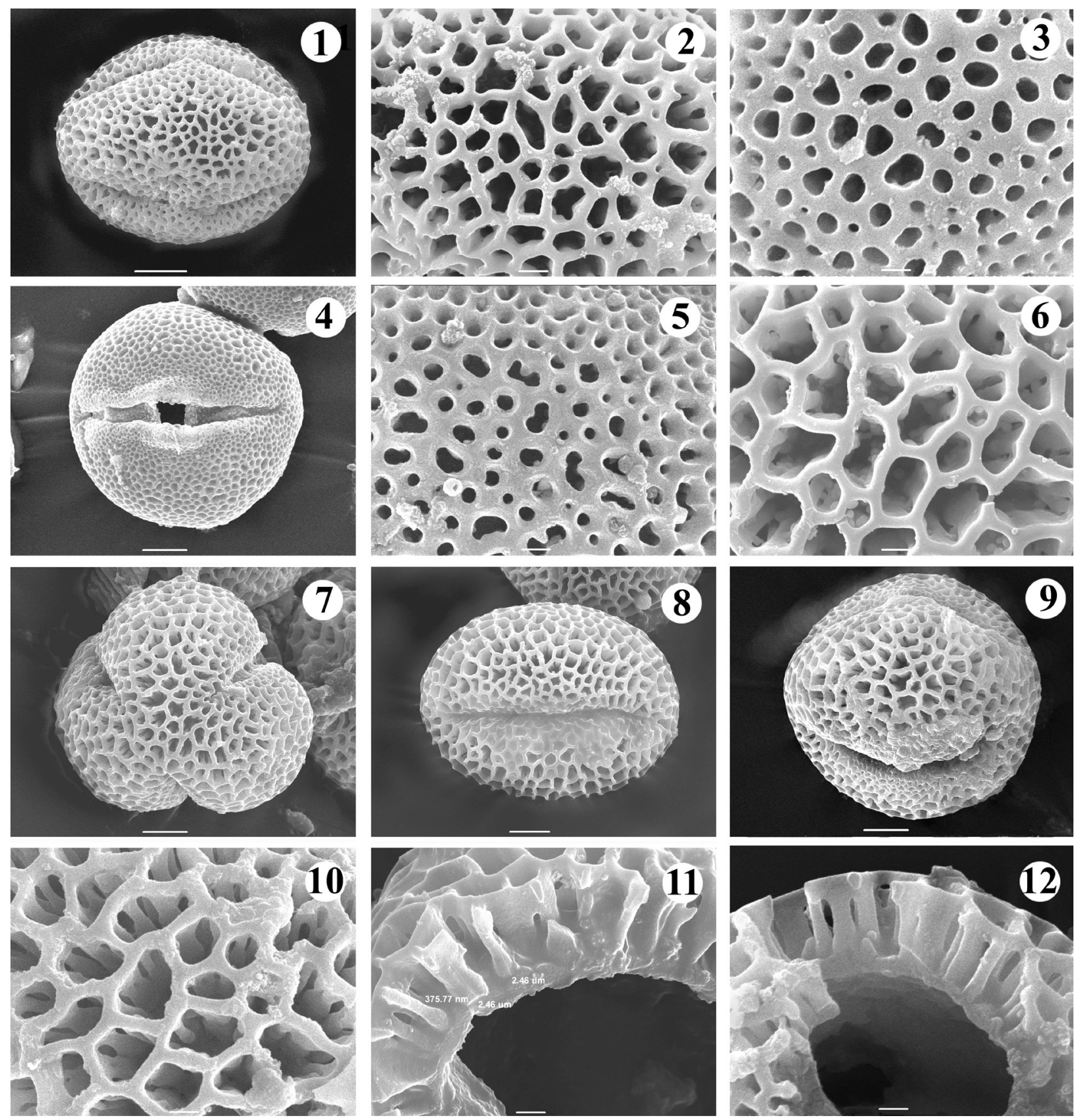

Fig. 6. Pollen general view $(1,4,7-9)$, pollen ornamentation $(2,3,5,6,10)$ sporoderm cleavage $(11,12)$ of Euonymus species (SEM) 1, 2-E. obovatus; $3-$ E. americanus; 4, 5-E. yesoensis; 6-E. oxyphyllus; 7, $10-$ E. miniatus; 8, 11 - E. planipes; 9, 12 - E. sachalinensis. Scale bar - $5 \mu \mathrm{m}(1,4,7-9), 1 \mu \mathrm{m}(2,3,5,6,10.11,12)$. 
4-6; Fig. 3: 2, 3; Fig. 5: 1-5, 7, 8, 10-12; Fig. 6: 11, 12). The exine in the center of mesocolpium or/ and apocolpium is usually thickened by columellae elongation. The columellae are clear, smooth, and often long, the distance between them (each to the other) is equal or slightly smaller than their thickness. The tectum and the foot layer (with endexine) are usually not thick, but approximately equal each other in thickness. The thickest exine layer is columellaete infratectum, as it is often more than twice (rarely the same thickness) of the tectum or foot layer wideness. CLSM data show the same distribution of the extexine layers, whereas the border of the endexine and the foot layer is not visible with SEM and CLSM.

\section{Discussion}

\section{Palynomorphological features}

According to palynomorphological data the genus Euonymus as the other genera of the family Celastraceae, is quite homogeneous. The pollen is represented by 3-colporate type with reticulate variations of surface ornamentation (Lobreau, 1969; Perveen, Qaiser, 2008). The pollen morphology of all studied species is very uniform, all the characteristics studied have a small range of variability. However, morphological features of some species have been revealed both in the structure of apertures and in exine ornamentation.

We observed parallel to the colpi exine thinning by LM. Similar thinnings have been found in other angiosperm families, such as Crassulaceae, Araliaceae, Linaceae, by our unpublished data. CLSM studies confirmed both sides have exine thinnings parallel to the colpus, which in the combination with ora, looks like H-shaped endoaperture (Fig. 4: 6, 9; Fig. 5). The TEM investigation of Celastraceae sporoderm was conducted by D. Lobreau-Callen and B. Lugardon (1972-1973) on an example of the exine of both acetolized and non-acetolized grains of $E$. europaeus, E. japonicus and Celastrus orbiculatus. The endexine structure has been illustrated, and the authors showed that thinning parallel to the colpi is formed in endexine.

Variations in the number and arrangement of apertures were found in several specimens. This article provides a quantitative content of atypical pollen grains in the studied specimens. The deviant pollen forms are widely distributed in flowering plant taxa. It has been found that, as in many flowering plant taxa, pollen forms with different aperture arrange- ment may be arranged in continuous regular series (Pozhidaev, 2009), in which, from form to form, the aperture position changes smoothly and continuously to follow a regular geometric pattern, that demonstrates non-specificity at a high taxonomic level (families, orders and subclass of dicotyledons and above; compare Fig. 4 of this article and figures in the paper of A. Pozhidaev (2009)). I. Dajoz et al. $(1991,1993)$ showed that 3-aperturate and 4-aperturate pollen grain of heteromorphic species Viola diversifolia differ by germination and longevity. In Viola calcarata the ratio of pollen with four and five apertures is variable according to altitude (TillBottraud et al., 1999). The authors (Till-Bottraud et al., 2001) supposed pollen heteromorphism to be an evolutionarily stable strategy. C. Prieu et al. (2016) have investigated the connection between aperture patterns and harmomegathy in the pollen of wild type and three Arabidopsis thaliana mutants with zero, four, or a mix of four to eight apertures, and have suggested that "pollen grains with few apertures survive better than pollen with many apertures". A. Mignot et al. (1994), S. Nadot et al. (2000), R. Humphrey (2016) and our unpublish data show that some angiosperm taxa have deviated pollen forms, but the others have only typical pollen grains.

The most common type of ornamentation of the Euonymus pollen is reticulate, a few parts of the species have microreticulate or perforate pollen surface or sometimes we found heterobrochate surfaces (Fig. 2).

We can't group species by pollen characteristics, because we found many intermediate types such as in pollen size or in endoaperture or surface structure. Endoapertures differ by size from gross, clear up to small, unclear; exine thinning side by side to colpi are found from observable to poor distinguishable; the ornamentation varies by lumina and muri sizes.

The most characteristic features of different species which help us to tell the difference between species by pollen grains are size and form of endoapertures and pollen ornamentation. These provide an opportunity to use palynomorphological data in pollen-spore analysis and clarify some taxonomical questions.

\section{Pollen morphology for systematics}

The genus is divided into two subgenera ( $E u$ onymus and Kalonymus) and from 4 to 12 sections. Subgenus Kalonymus is recognized the most recent group of Euonymus. There is no pollen with H-like 
ora (except 2 species) and there are significantly more common species with deviated pollen forms in this subgenus.

J.-S. Ma (2001) has realized the last world revision of the genus and has reduced the number of sections up to 4 in the subgenus Euonymus and has not find divisions within the subgenus Kalonymus. The number of species was reduced to 129 , as more than 50 species were considered as synonymous.

After J.-S. Ma (2001) the subgenus Kalonymus includes 16 species. These species are classified into Kalonymus by other authors (Blakelock, 1951; Leonova, 1974; Tzvelev, 2004; Savinov, Baikov, 2007) too. We have studied the pollen of 16 species from the subgenus, but J. S. Ma (2001) names some of them as synonymous of these investigated specimens (E. dasydictyon, E. miniatus, E. planipes, E. porphyreus, E. yesoensis). E. maximowiczianus is not noted in this system (only Kalonymus maximowicziana Prokh. as synonym of E. sachalinensis).

J.-S. Мa (2001) considers E. dasydictyon и $E$. porphyreus as synonymous of $E$. frigidus, as the first two taxa belong to different series of T. Leonova systems (1974), and the third taxon refers to the other section by T. Leonova (1974) and R. A. Blakelock (1951). Pollen of E. dasydictyon and E. porphyreus is very similar, but grains of $E$. porphyreus have larger ora and smaller lumina in the surface ornamentation. Grains of E. frigidus are smaller in size, mainly oblate, verrucate-reticulate ornamentation, which is a unique type in the genus, and the exine is more than twice thin. Palynomorprological data confirms the separation of this species. Molecular data (Li et al., 2014) also contradicts the association of E. porphyreus and E. frigidus in one species.

E. yesoensis is referred to synonyms for E. oxyphyllus, which is consistent with R. A. Blakelock's (1951) and T. Leonova's (1974) systems, and is not comport with palynological data. Pollen of these species has different ornamentation - reticulate with lumina from $3.0 \mu \mathrm{m}$ in E. oxyphyllus and reticulateperforate in E. yesoensis (Fig. 6: 4-6).

$E$. miniatus and $E$. planipes are synonymous of E. sachalinensis (Fig. 6: 7-12). Pollen grains of these taxa do not differ. Pollen morphology has allowed these taxa to combine in one species.

R. A. Blakelock (1951) and T. Leonova (1974) classified E. yakushimensis in the subgenus Kalonymus, but J.-S. Ma (2001) included this species in the subgenus Euonymus (section 4 Ilicifolia). According to the palynological characteristics this species is like to representative of section Ilicifolia: E. yakushimensis grains have distinctive $\mathrm{H}$-shaped aperture and reticulate-perforate surface; but the species of the subgenus Kalonymus have a very rare $\mathrm{H}$-shaped ora, and the surface is mainly reticulate with the lumina more than $2 \mu \mathrm{m}$.

In general, the pollen of most studied species of the subgenus Kalonymus can be attributed to one group of pollen grains with reticulate often with a big size of lumina sculpture and a clearer ora. Pollen of E. frigidus and E. yesoensis, discussed above, as well as pollen of E. cornutus, with distinctive H-shaped aperture (Fig. 5: 2), are segregated from this group. It is impossible to separate the subgenus species by pollen features series, which were established in T. Leonova (1974) and I. Savinov, K. Baikov (2007) systems. Although recent studies (Simmons et al., 2012; Li et al., 2014; Sidorov, Trusov, 2015; Sidorov et al., 2014) confirmed the isolation of the subgenus, the main group of Kalonymus pollen may also include pollen of a large number of species of another subgenus.

J.-S. Ma (2001) divided the subgenus Euonymus into 4 sections. The section Echinococcus is represented by 18 species. We studied the pollen of 5 specimens of this section, including E. obovatus that J.-S. Ma (2001) recognized as synonyms of $E$. americanus. Pollen of these species differs both in structure/size of ora and thickness of muri, so $E$. obovatus has a reticulate exine surface with narrow muri, but E. americanus has a reticulate-perforate ornamentation with rather thick muri (on the verge of a perforated) (Fig. 6: 1-3). R. A. Blakelock (1951), T. Leonova (1974) and I. Savinov, K. Baikov (2007) splitted this section into two series, what have not agreed with the research of Y-Ch. Zheng et al. (2012). Neither the complexity of palynological features, none any one of the regular features (size, structure apertures, exine structure, surface type) have not confirmed the division of the section Echinococcus into the series, as well as the isolation of this section within the genus and the affinity to the subgenus Kalonymus, that has been affirmed by recent molecular studies (Li et al., 2014).

The Melanocarya section consists of 11 species. Four of the studied pollen samples belong to 2 species by J.-S. Ma (2001), E. sacrosanctus and E. subtriflorus referred to synonyms of E. alatus. T. Leonova (1974) believed that E. subtriflorus is synonymous with E. alatus. Although pollen of E. subtriflorus is a little smaller sized, the differences of pollen grains among these three taxa are inconsiderable, it is permissible to recognize their association, the similarity of E. sacrosanctus and E. alatus as confirmed by R. Sidorov, N. Trusov (2015). The 
fourth our specimen of the section $-E$. pendulus (Fig. 2: 10; Fig. 3: 7; Fig. 4: 8-10) is described by J.-S. Ma (2001) as synonym of E. lucidum D. Don; we have not studied E. lucidum and have not made a comparison with synonym. E. salicifolius $(=$ E. lawsonii var. salicilolius) is included in Melanocarya section by R. A. Blakelock (1951) and in Euonymus section by J.-S. Ma (2001). A distinctive set of features of pollen of this species was not detected, the pollen grains are medium-sized $24-32 \mu \mathrm{m}$ in diameter, with heterobronchate surface and wellexpressed H-liked apertures. The above-described pollen grains also are found in E. pendulus (section Melanocarya) and in part of the representatives of the Euonymus section.

The Ilicifolia section includes 20 species. We investigated 6 pollen samples. E. patens (Fig. 5: 7) is synonymous with $E$. hederaceus, and it is confirmed by palynomorphological data. Pollen of Ilicifolia species has well-expressed, H-shaped apertures (except $E$. benthamii), perforate or reticulate-perforate (diameter of lumina less than $1.3 \mu \mathrm{m}$ ), and often heterobronchate exine surfaces. The section is considered close to the primitive form. I. Savinov, K. Baikov (2007) discussed the possibility of Ilicifolia location as a separate subgenus. R. Sidorov, N. Trusov (2015) believed that this allocation is acceptable, as they found that the ratio of the LCD-composition lipid seeds and aril of E. japonicus differs from those characteristics of the other species of the genus. However, this study comprises of only one species from the section and the pollen of E. japonicus, which has the rare feature - 3-angular outline in polar view and is distinct in the complex of palynomorphological characteristics. The pollen of the other species of the section is more typical for the genus.

Euonymus is the largest section of the subgenus Euonymus; it includes 64 species, about a half of the genera species according to J.-S. Ma (2001). We have investigated 31 pollen specimens from this section, 12 samples (E. czernjaevii, E. moldavicus, $E$. velutinus, E. bungeanus, E. integerrimus, E. lanceifolius, E. pauciflorus, E. juzepczukii, E. sieboldianus, E. vidalii, E. yedoensis var. koehneana, E. przewalskii) are reduced to synonyms by J.-S. Ma (2001).

All these investigated species, except $E$. salicifolius and E. grandiflorus, belong to 4 series of the section Biloculares according to R. A. Blakelock (1951). Application of pollen data to taxonomy of $E$. salicifolius is discussed in a paragraph of the section Melanocarya. E. grandiflorus is a species of section
Multiovulatus by R. A. Blakelock (1951), T. Leonova (1974), I. Savinov, K. Baikov (2007). Pollen of E. grandiflorus (Fig. 4: 13-15) is characterized by quite large sizes, $32-37 \mu \mathrm{m}$ in diameter.), reticulate surface, small-sized ora. In E. grandiflorus we have found the most number of deviated pollen grains (50 $\%)$ in the genus. The division on section and series of systems of R. A. Blakelock (1951), T. Leonov (1974), and I. Savinov, K. Baikov (2007) on the basis of pollen data is not confirmed.

We noted that the most diverse and distinctive pollen features were found in the representatives series Myrianthi (section Biloculares). These characteristics are the next: polar view of E. gibber, E. laxiflorus, E. nitidus grains is 3 -angular (Fig. 1: 4, Fig. 4: 1, 2), pollen of $E$. nitidus have minimal sizes $17-22 \mu \mathrm{m}$ in diameter, pollen of E. dichotomus and E. myriantus is small too, no more than 25 $\mu \mathrm{m}$ in diameter; three representatives ( $E$. dichotomus, E. myriantus, E. nitidus) from all subgenus Euonymus have perforate exine ornamentation (Fig. 2: 12); (exine ornamentation of E. glaber (Fig. 2: 9 ) is reticulate-perforate and reticulate in E. gibber, E. laxiflorus (Fig. 2: 8), E. tingens); on apocolpium lumina in E. glaber is more than mesocolpium lumina size. I. Savinov, K. Baikov's (2007) brings three sections Ilicifolia, Multiovulatus and Myrianthus of primitive spindle trees together in the group; this group is the most diverse from a palynomorphological point of view. Exine surface in this group vary from perforate and microreticulate up to reticulate with small-sized lumina, but large-sized lumina is not a commonly found.

As for synonyms of the species mentioned in the section Euonymus J.-S. Ma (2001) they refer to the section Biloculares series Lophocarpi and Pseudovyenomi by R. A. Blakelock (1951). According to J.-S. Ma (2001) E. europaeus (Fig.5: 4) includes $E$ czernjaevii, E moldavicus and E. velutinus. T. Leonova (1974) did not recognize E. moldavicus as separate species, while the other three taxa put together in the Lophocarpae series of the section Euonymus.

J.-S. Ma (2001) classified E. juzepczukii, E. sieboldianus, E. lanceifolius, E. vidalii, E. yedoensis var. koehneana into E. hamiltonianus. After R. A. Blakelock (1951) E. sieboldianus, E. lanceifolius, E. yedoensis var. koehneana also are synonyms of $E$. hamiltonianus. Pollen grains of E. juzepczukii, E. lanceifolius, E. sieboldianus, E. hamiltonianus are from 23 to $34 \mu \mathrm{m}$ in diameter, ora are more or less expressed from 2 to $5 \mu \mathrm{m}$ in diameter, the surface is mainly reticulate with large lumina sizes, columellas and the bottom of lumina 
are often visible. Near colpa, the size of lumina decreases, on the apocolpium lumina usually are equal or less than on the mesocolpium, exine from 2.2 to $4.0 \mu \mathrm{m}$ thick, columellas are clear and long. Grains of this group are similar among themselves, so association is allowed. R. A. Blakelock (1951) put E. maackii to E. hamiltonianus var. maackii and J.S. Ma (2001) represents E. bungeanus as E. maackii synonym. E. maackii grains belong to the above described pollen group, they are very similar to those at $E$. bungeanus. Distinctive features of pollen of two samples are quite substantial. Grains are larger usually from $30 \mu \mathrm{m}$ in the diameter, ora large, more than $4-7 \mu \mathrm{m}$ in the diameter, a surface is reticulate perforate or reticulate, and there is an increase of the lumina sizes on an apocolpium in comparison with mesocolpium.

E. semenovii and E. przewalskii were united by J.-S. Ma (2001) in one species, while T. Leonova (1974) and I. Savinov, K. Baikov (2007) believed these two species can be treated in one series. Its pollen is also poorly distinguishable, within morphological fluctuations into one species.

E. integerrimus and E. pauciflorus were reduced by J.-S. Ma (2001) to E. verrucosus as synonyms. T. Leonova (1974) and I. Savinov, K. Baikov (2007) relegated these to one series. Their pollen is very similar, its association is admissible.

N. Tzvelev (2004) has allocated E. nanus into one new section Nanevonymus. N. Tzvelev (2004) didn't divide the genus into subgenera and considered Kalonymus as one section. All spindle trees of Eastern Europe have been united in 8 sections. Pollen of E. nanus is typical for the genus.

The species which pollen is similar by some characteristics as well as on their complex, often belong to different sections and subgenera. Palynological data do not confirm modern taxonomical systems.

The reduction of some taxa (E. obovatus $=E$. americanus; $E$. dasydictyon $=E$. frigidus; E. porphyreus $=$ E. frigidus; E. yesoensis $=$ E. oxyphyllus; E. czernjaevii $=$ E. europaeus; E. velutinus $=$ E. europaeus; E. vidalii $=$ E. hamiltonianus; $E$. yedoensis var. koehneana $=E$. hamiltonianus) to synonyms is not confirmed palynologically.

But the next species $(E$. planipes $=E$. sachalinensis; E. miniatus $=$ E. sachalinensis; E. molda- vicus $=$ E. europaeus; E. sacrosanctus $=$ E. alatus; E. subtriflorus $=E$. alatus; E. patens $=E$. hederaceus; E. bungeanus $=$ E. maackii; E. przewalskii $=$ E. semenovii; E. juzepczukii $=E$. hamiltonianus; E. sieboldianus $=E$. hamiltonianus) can be synonyms by pollen data.

\section{Conclusion}

3-colporate, reticulate pollen of representatives of the genus Euonymus is typical for the family Celastraceae as a whole.

No correspondence of palynomorphological data to the accepted sectional subdivision of subgenus Euonymus (Ma, 2001) has been found. In addition, no corresponding data have been discovered on taxonomic groups isolated within the genus according to R. A. Blakelock (1951), T. Leonova (1974), N. Tsvelev (2004) and I. Savinov and K. Baikov (2007), neither by complex of pollen characters nor by one of them separately (the shape of the pollen grain, the structure of the apertures, the structure of the exine, surface sculpture, dimensions).

The species which pollen is similar by some characteristics as well as on their complex often belong to different sections and subgenera. The palynological data do not confirm modern taxonomical systems.

The palynomorphological data do not confirm the status of Kalonymus neither as a subgenus, nor a separate genus.

The reduction of half species into synonyms is not proved by pollen morphology.

Nevertheless, the pollen peculiarities of certain species are allowed in order to use the obtained data in the pollen-spore analysis.

\section{Acknowledgements}

The study was carried out within the framework of institutional research project No. 01201255609 of the Komarov Botanical Institute of the Russian Academy of Sciences. The research was done using equipment of The Core Facilities Center "Cell and Molecular Technologies in Plant Science" at the Komarov Botanical Institute RAS (St. Petersburg, Russia).

\section{REFERENCES / ЛИTEPATУPA}

Artyushenko A. T., Romanova L. S. 1984. Morphologiya pyltsi reliktovykh, endemichnykh i redkich vidov flori Ukrainy [Pollen morphology of relic, endemic and rare species of Ukraine flora]. Naukova dumka, Kiev, 48 pp. [In Russian]. (Артюшенко A. T., Романова Л. С. Морфология пыльцы реликтовых, эндемичных и редких видов флоры Украины. Киев: Наукова думка, 1984. 48 с.). 
Blakelock R. A. 1951. A synopsis of the genus Euonymus L. Kew Bull. 2: 210-290.

Chester P. I., Raine J. L. 2001. Pollen and spore keys for Quaternary deposits in the northern Pindos Mountains, Greece. Grana 40: 299-387. DOI: 10.1080/00173130152987535

Dajoz I., Till-Bottraud I., Gouyon P. H. 1991. Evolution of pollen morphology. Science 253: 66-68. DOI: 10.1126/ science.253.5015.66

Dajoz I., Till-Bottraud I., Gouyon P. H. 1993. Pollen aperture polymorphism and gametophyte performance in Viola diversifolia. Evolution 47: 1080-1093. DOI: 10.1111/j.1558-5646.1993.tb02137.x

Erdtman G. 1952. Pollen morphology and plant taxonomy: Angiosperms. Stokholm, 539 pp.

Gavrilova O. A. 2014. Application of confocal laser scanning microscope for pollen wall morphology study. Bot. Zhurn. (Moscow \& St. Petersburg) 99(10): 1139-1147 [In Russian]. (Гаврилова О. А. Применение конфокальной лазерной сканирующей микроскопии (КЛСМ) для исследования морфологии оболочки пыльцевых зерен // Бот. журн., 2014. Т. 99, № 10. С. 1139-1147).

Hesse M., Halbritter H., Weber M., Buchner R., Frosch-Radivo A., Ulrich S., Zetter R. 2009. Pollen terminology. Springer-Verlag, Wien, 266 pp. DOI: 10.1007/978-3-211-79894-2

Heusser C. J. 1971. Pollen and spores of Chili. Modern types of Pteridophyta, Gymnospermae and Angiospermae. Tuczon, $167 \mathrm{pp}$.

Huang T.-C. 1972. Pollen flora of Taiwan. Taipei, 297 pp.

Humphrey R. P. 2016. Pollen heteromorphism is pervasive in Thalictrum (Ranunculaceae). Plant Systematics and Evolution 302(8): 1171-1177. DOI: 10.1007/s00606-016-1312-8

Ikuse M. 1956. Pollen grains of Japan. Tokyo, 279 pp.

Kupriyanova L. A. 1972. Celastraceae Batsch. In: Kupriyanova L. A., Aleshina L. A. Pyltsa dvudolnykh rasteniy flory yevropeyskoy chasti SSSR [Pollen of dycotiledon plants of flora of European part of URSS]. Vol. 1. Leningrad, 86-89 pp. [In Russian]. (Куприянова Л. А. Сем. Celastraceae Batsch // Куприянова Л. А., Алёшина Л. А. Пыльца двудольных растений флоры европейской части СССР. Т. 1. Л., 1972. С. 86-89).

Leonova T. G. 1974. Beresklety SSSR i sopredelnykh stran [Spindle trees of USSR and neighboring countries]. Nauka, Leningrad, 132 pp. [In Russian]. (Леонова T. Г. Бересклеты СССР и сопредельных стран. Л.: Наука, 1974. 132 c.).

Li Y.-N., Xie L., Li J.-Y., Zhang Z.-X. 2014. Phylogeny of Euonymus inferred from molecular and morphological data. J. Syst. Evol. 52(2): 149-160. DOI: 10.1111/jse.12068

Lobreau D. 1969. Les limites de 1'"ordre" des Célastrales d'après le pollen. Pollen et spores 11(3): 499-555.

Lobreau-Callen D., Lugardon B. 1972-1973. L' aperture a repli du pollen des Celastraceae. Nat. Monspel. Ser. Bot. Fasc. 23-24, I-II: 205-210.

Loesener Th. 1942. Celastraceae. In: Die Natürlishen Pflanzenfamilien. Vol. 20B. Eds. A. Engler, K. Prantl. Wilhelm Engelmann, Leipzig, Berlin, 87-197 pp.

Ma J.-S. 2001. A revision of Euonymus (Celastraceae). Thaiszia - J. Bot., Košice 11: 1-264. URL: https://www. upjs.sk/public/media/5743/thaiszia-11-1-264-2001-ma.pdf

Ma J.-S., Funston A. M. 2008. Celastraceae, Euonymus. In: Flora of China. Vol. 11 (Oxalidaceae through Aceraceae). Eds. Z. Y. Wu, P. H. Raven, D. Y. Hong. Science Press, Beijing, and Missouri Botanical Garden Press, St. Louis, 440-463 pp.

Mignot A., Hoss C., Dajoz I., Leuret C., Henry J. P., Dreuillaux J. M., Heberle-Bors E. et al. 1994. Pollen aperture polymorphism in the angiosperms: importance, possible causes and consequences. Acta Bot. Gallica 141: 109-122. DOI: 10.1080/12538078.1994.10515144

Nadot S., Ballard H. E., Creach J. B., Dajoz I. 2000. The evolution of pollen heteromorphism in Viola: a phylogenetic approach. Pl. Syst. Evol. 223: 155-171. DOI: 10.1007/bf00985276

Nair P. K. K. 1965. Pollen grains of Western Himalayan plants. Asia Publ. H., London, 102 pp.

Nakai T. 1941. Subdivisions of the genus Euonymus. J. Jap. Bot. 17: 615-619.

Nakamura J. 1980. Diagnostic characters of pollen grains of Japan. Spec. Publ. Osaka Mus. Nat. Hist. $12:$ 1-157.

Perveen A., Qaiser M. 2008. Pollen flora of Pakistan. LVIII. Celastraceae. Pak. J. Bot. 40(3): 957-962.

Poliychuk Yu. S. 1978. About pollen of Far East spindle trees. In: Redkiye $i$ ischezayushchiye rasteniya yuga Dalnego Vostoka (biologiya, ekologiya, kariologiya) [Rare and disappearing plants of the south of the Far East (biology, ecology, karyology)]. Vladivostok, 142-144 pp. [In Russian]. (Полийчук Ю. С. О пыльце дальневосточных бересклетов // Редкие и исчезающие растения юга Дальнего Востока (биология, экология, кариология). Владивосток, 1978. С. 142-144).

Pozhidaev A. E. 2009. The structure of the diversity of the morphological character on the example of the location of pollen apertures in flowering plants and the natural ordering of biological diversity, or that such a variety (ways of describing and interpreting). In: Trudy Zoologicheskogo instituta RAN. Prilozheniye 1. [Перевод на англ.] 150-182 pp. [In Russian]. (Пожидаев $\boldsymbol{A}$. $\boldsymbol{E}$. Структура многообразия морфологического признака на примере расположения апертур пыльцы цветковых и естественная упорядоченность биологического многообразия, или что 
такое многообразие (способы описания и интерпретации) // Труды Зоол. института РАН. Приложение № 1 , 2009. C. 150-182).

Praglowski J., Punt W. 1973. An elucidation of the microreticulate structure of the exine. Grana Palynol. 13: 45-50.

Premathilake R., Nilsson S. 2001. Pollen morphology of endemic species of the Horton Plains National Park, Sri Lanka. Grana 40: 256-279. DOI: 10.1080/00173130152987508

Prieu C., Matamoro-Vidal A., Raquin C., Dobritsa A., Mercier R., Goyon P.-H., Albert B. 2016. Aperture number influences pollen survival in Arabidopsis mutants. Am. J. Bot. (Special Issue on the Ecology and Evolution of Pollen Performance) 103: 452-459. DOI: 10.3732/ajb.1500301

Prokhanov Ya. I. 1949. Celastraceae Lindl. In: Flora SSSR [Flora of USSR]. Vol. 14. Publishers of Academy of Sciences of USSR, Moscow, Leningrad, 552-577 pp. [In Russian]. (Проханов Я. И. Celastraceae Lindl. // Флора СССР. Т. 14. М.-Л.: АН СССР, 1949. С. 552-577).

Prokhanov Ya. I. 1960. Synopsis of Celastraceae system of USSR. Additions and changes. Botanicheskiye materialy Gerbariya Botanicheskogo instituta AN SSSR [Botan. mater. Herb. Bot. In-ta of Academy of Sciences of USSR] 20: 409-412 [In Russian]. (Проханов Я. И. Конспект системы бересклетовых СССР. Добавления и изменения // Ботан. матер. Герб. Бот. ин-та АН СССР, 1960. Т. 20. С. 409-412).

Punt W., Hoen P. P., Blackmore S., Nilsson S., Le Thomas A. 2007. Glossary of pollen and spore terminology. Rev. Palaeobot. Palyn. 143(1-2): 1-81. DOI: 10.1016/j.revpalbo.2006.06.008

Savinov I. 2007. Some morphological basics for a revision of the tribe Euonymeae Loes. (Celastraceae R. Br.). Wulfenia 14: 97-104.

Savinov I. A., Baikov K. S. 2007. The analysis of phylogenetic relations in the genus Euonymus (Celastraceae R. Br.) using SYNAP method. Turczaninowia 10, 3-4: 36-50 [In Russian]. (Савинов И. А., Байков К. С. Анализ филогенетических связей в роде Euоnymus L. (Celastraceae R. Br.) с помощью метода SYNAP // Turczaninowia, 2007. Т. 10, вып. 3-4. С. 36-50).

Schulz B. 2006. Studien zu den Früchten und Semen ausgewählter Euonymus - Arten. Mitt. Dtsch. Dendrol. Ges. 91: $127-145$.

Shimanovich E. I. 1987. Beresklet [Spindle tree]. Moscow, 64 pp. [In Russian]. (Шиманович Е. И. Бересклет. M., 1987. 64 c.).

Shimakura M. 1973. Palinomorfs of Japanese plants. Spec. Publ. Osaka Mus. Nat. Hist. 5: 1-60.

Sidorov R. A., Zhukov A. V., Pchelkin V. P., Vereshchagin A. G., Tsydendambaev V. D. 2014. Content and Fatty Acid Composition of Neutral Acylglycerols in Euonymus Fruits. J Am Oil Chem Soc. 91(5): 805-814. DOI: 10.1007/ s11746-014-2425-2

Sidorov R. A., Trusov N. A. 2015. On the possibility of phylogenetic relationship in the genus Euonymus in comparison with the data on the LC composition of mature fruit triacylglycerols. In: "Semicentenary after Konstantin Meyer": XIII Moscow Symposium on Plant Phylogeny: Proceedings of the International Conference (February 2-6, 2015, Moscow). Moscow, 280-284 pp. [In Russian]. (Сидоров Р. А., Трусов Н. А. О возможности привлечении к решению вопроса филогенетических отношений в роде Euonymus данных ЖК-состава триацилглицеринов ариллусов зрелых плодов // Материалы междунар. конф. ХІІІ Московского совещания по филогении растений «50 лет без К. И. Мейера». М., 2015. С. 280-284.)

Simmons M. P., McKenna M. J., Bacon C. D., Yakobson K., Cappa J. J., Archer R. H., Ford A. J. 2012. Phylogeny of Celastraceae tribe Euonymeae inferred from morphological characters and nuclear and plastid genes. Mol. Phylogenet. Evol. 62, 1: 9-20. DOI: 10.1016/j.ympev.2011.08.022

Till-Bottraud I., Gouyon P. H., Venable D. L., Godelle B. 2001. The number of competitors providing pollen on a stigma strongly influences intraspecific variation in number of pollen apertures. Evolutionary Ecology Research 3 : 231-253.

Till-Bottraud I., Vincent M., Dajoz I., Mignot A. 1999. Pollen aperture heteromorphism. Variation in pollen-type proportions along altitudinal transects in Viola calcarata. Comptes Rendus de l'Académie des Sciences de Paris, Sciences de la Vie 322: 579-589. DOI: 10.1016/S0764-4469(00)88528-5

Tzvelev N. N. 2004. Celastraceae. In: Flora Vostochnoy Yevropy [Flora of Eastern Europe]. Vol. 11. Moscow \& St. Petersburg, 437-449 pp. [In Russian]. (Цвелев Н. Н. Celastraceae // Флора Восточной Европы. Т. ХІ. М.-СПб., 2004. C. 437-449).

Wang F. N., Chein N. F., Chzang T. T. 1960. Morphology of pollen of Chinese plants. Beijing, 276 pp. [In Chinese].

Zheng Yan-Chao, Mu Xian-Yun, Li Yan-Nan, Tu Qiang, Zhang Zhi-Xiang. 2012. A Numerical taxonomic study of the Euonymus section Echinococcus (Celastraceae). Acta Bot. Yunnanica 3: 271-286. DOI: 10.3724/ SP.J.1143.2012.11183 